\title{
The RXLR Effector PcAvh1 Is Required for Full Virulence of Phytophthora capsici
}

\author{
Xiao-Ren Chen, ${ }^{1,2,+}$ Ye Zhang, ${ }^{1}$ Hai-Yang Li, ${ }^{3}$ Zi-Hui Zhang, ${ }^{1}$ Gui-Lin Sheng, ${ }^{1}$ Yan-Peng Li, ${ }^{1}$ \\ Yu-Ping Xing, ${ }^{1}$ Shen-Xin Huang, ${ }^{1}$ Hang Tao, ${ }^{1}$ Tung Kuan, ${ }^{2}$ Yi Zhai, ${ }^{2}$ and Wenbo $\mathrm{Ma}^{2}$ \\ ${ }^{1}$ College of Horticulture and Plant Protection, Yangzhou University, Yangzhou, Jiangsu Province 225009, China \\ ${ }^{2}$ Department of Microbiology and Plant Pathology, University of California, Riverside, CA 92521, U.S.A. \\ ${ }^{3}$ College of Plant Protection, Nanjing Agricultural University, Nanjing, Jiangsu Province 210095, China
}

Accepted 22 February 2019.

\begin{abstract}
Plant pathogens employ diverse secreted effector proteins to manipulate host physiology and defense in order to foster diseases. The destructive Phytophthora pathogens encode hundreds of cytoplasmic effectors, which are believed to function inside the plant cells. Many of these cytoplasmic effectors contain the conserved $\mathbf{N}$-terminal RXLR motif. Understanding the virulence function of RXLR effectors will provide important knowledge of Phytophthora pathogenesis. Here, we report the characterization of RXLR effector PcAvh1 from the broad-host range pathogen Phytophthora capsici. Only expressed during infection, $P c A v h 1$ is quickly induced at the early infection stages. CRISPR/Cas9-knockout of PcAvh1 in $P$. capsici severely impairs virulence while overexpression enhances disease development in Nicotiana benthamiana and bell pepper, demonstrating that PcAvh1 is an essential virulence factor. Ectopic expression of PcAvh1 induces cell death in $N$. benthamiana, tomato, and bell pepper. Using yeast two-hybrid screening, we found that PcAvh1 interacts with the scaffolding subunit of the protein phosphatase 2A (PP2Aa) in plant cells. Virus-induced gene silencing of $P P 2 A a$ in $N$. benthamiana attenuates resistance to $P$. capsici and results in dwarfism, suggesting that PP2Aa regulates plant immunity and growth. Collectively, these results suggest that $P c A v h 1$ contributes to $P$. capsici infection, probably through its interaction with host PP2Aa.
\end{abstract}

The gene sequences reported by this study have been submitted to the National Center for Biotechnology Information database with accession numbers MF975713, MF996339, and MF996340.

${ }^{\dagger}$ Corresponding author: X.-R. Chen; xrchen@yzu.edu.cn

Funding: This work was financially supported by the National Natural Science Foundation of China (grant number 31671971, 31871907), Natural Science Foundation of Yangzhou City (China) (grant number YZ2016121), the Special Fund for Agro-Scientific Research in the Public Interest of China (grant number 201303018), the Yangzhou University 2016 Project for Excellent Young Key Teachers, Postgraduate Research \& Practice Innovation Program of Jiangsu Province (SJCX19_0895), and High-Level Talent Support Program of Yangzhou University to X.-R. Chen.

*The $\boldsymbol{e}$-Xtra logo stands for "electronic extra" and indicates that six supplementary figures and two supplementary tables are published online.

The author(s) declare no conflict of interest.

(c) 2019 The American Phytopathological Society
Keywords: bell pepper, CRISPR/Cas9, oomycete effectors, Phytophthora capsici, Phytophthora diseases, plant immunity, protein phosphatase 2A, RXLR effector, virulence

In nature, plants encounter attacks by numerous microbial pathogens and have evolved a two-tier immune system to protect themselves from infection (Dou and Zhou 2012; Jones and Dangl 2006). The first layer depends on the perception of one or more pathogen-associated molecular patterns (PAMPs) or microbe- and damage-associated molecular patterns by pattern recognition receptors at the plant plasma membrane, resulting in PAMP-triggered immunity (PTI) (Couto and Zipfel 2016). However, host-adapted pathogens have developed strategies to suppress PTI by delivery of an array of virulence proteins, collectively known as effectors. As a defense response, the second layer of plant immunity is activated after direct or indirect recognition of pathogen effectors by the structurally conserved disease resistance $(\mathrm{R})$ proteins as intracellular receptors. This specific recognition, namely effector-triggered immunity (ETI), often involves the hypersensitive response (HR) with a hallmark of a rapid, localized programmed cell death (PCD) (Jones and Dangl 2006). Effectors recognized by plant resistance $(\mathrm{R})$ proteins are termed avirulence (Avr) factors. However, pathogens have also evolved strategies to suppress ETI. This coevolutionary arms race continues between pathogens and their plant hosts (Ma and Guttman 2008).

One of the major tasks in plant pathology is to understand the contribution of pathogen effectors in disease development and the underlying mechanisms. Species of genus Phytophthora are some of the most aggressive plant pathogens (Erwin and Ribeiro 1996). For example, P. infestans is the causal agent of the potato late blight disease, while $P$. sojae leads to the stem and root rot of soybean (Kamoun et al. 2015). To establish successful infection and colonization, Phytophthora spp. utilize two groups of secreted effectors, apoplastic effectors that act in the plant extracellular space and cytoplasmic effectors that are translocated into the plant cells (Kamoun 2006). Apoplastic effectors include elicitins, NLPs (necrosis- and ethylene-inducing peptide 1-like proteins), PcF/SCR (small cysteine-rich) proteins, CBEL (cellulose binding, elicitor, and lectin-like) proteins, and enzyme inhibitors (Kamoun 2006). Of the apoplastic effector arsenal, particular ones can be detected by plants as PAMPs to trigger PTI while some effectors that evade plant perception modulate plant apoplastic immunity to favor pathogen infection (Jones and Dangl 2006; Kamoun 2006; Wang and Wang 2018). 
Cytoplasmic effectors described so far include RXLR and CRN (Crinkler) protein families (Kamoun 2006). CRN effectors, named for their crinkling and necrosis-inducing activity, are composed of a highly conserved $\mathrm{N}$-terminal domain containing a signal peptide and an LXLFLAK motif, which mediates translocation into the host cell, and a diverse collection of C-terminal domains that carry effector functions (Amaro et al. 2017). The RXLR protein family is the largest class of translocated effectors, which includes all thusfar-identified Avr proteins (Anderson et al. 2015; Kamoun 2006). RXLR effectors possess a modular architecture including the $\mathrm{N}$ terminal signal peptide for protein secretion, the following RXLR motif for host translocation, and the diverse C-terminal domain executing virulence activity (Dou et al. 2008; Kamoun 2006; Win et al. 2007). Evolutionary selection has acted mainly on the Cterminal regions, probably due to the coevolution with plant target proteins (Anderson et al. 2015; Win et al. 2007). A conserved core $\alpha$-helical fold occurs in approximately $44 \%$ of annotated Phytophthora RXLR effectors, both as a single domain and in tandem repeats of up to 11 units (Boutemy et al. 2011). A large portion of RXLR effectors have the ability to suppress host one or both PTI and ETI (Anderson et al. 2015; Wang et al. 2011). So far, host proteins including the susceptibility factors targeted by a few Avrs and RXLR effectors have been identified (Anderson et al. 2015; Boevink et al. 2016; Du et al. 2015; King et al. 2014; Kong et al. 2017; Qiao et al. 2015; Yang et al. 2016). These studies show that Phytophthora RXLR effectors target a wide range of plant pathways at various levels to either suppress immunity, manipulate other processes, or both to benefit the colonization of the pathogens.

$P$. capsici is a destructive plant pathogen with a global distribution (Erwin and Ribeiro 1996; Lamour et al. 2012b). Unlike most Phytophthora species, which have narrow host ranges, $P$. capsici causes significant damage to the production of a wide variety of crops, such as pepper, tomato, watermelon, cucumber, pumpkin, and snap bean (Erwin and Ribeiro 1996; Kamoun et al. 2015; Lamour et al. 2012b). Worldwide vegetable production valued at well over one billion dollars is threatened each year (Lamour et al. 2012b). As the impact of crop loss due to $P$. capsici has been expanding, an increasing effort has been made to study the pathogenicity mechanisms of this poorly described pathogen. Bioinformatic analysis of the draft genome sequence of $P$. capsici revealed a large effector repertoire consisting of 357 RXLR effectors, 99 CRNs, and 42 NLPs (Feng et al. 2014; Lamour et al. 2012a). Localization and functional studies have led to the view that $P$. capsici CRN Ctermini execute their presumed effector function in the host nucleus, targeting processes required for immunity (Mafurah et al. 2015; Stam et al. 2013a and b; R. Stam, G. B. Motion, P. C. Boevink, and E. Huitema personal communication). Of 357 Avr homolog (Avh) genes, only one Avr3a-like gene (PcAvr3al) was characterized by using host-induced gene silencing in N. tabacum (Vega-Arreguín et al. 2014). Among the remainder, one (Pc129113, the Joint Genome Institute ID) was found to encode a RXLR effector of 478 amino acids, which shows $30.6 \%$ identity to the necrosis-inducing effector PsAvh365 of $P$. sojae (Chen et al. 2013) and is, thereby, designated PcAvh1. In this study, we characterized the virulence function of PcAvh1, which is highly expressed during in planta infection of Nicotiana benthamiana by P. capsici. Importantly, knockout (KO) of $P c A v h l$ in $P$. capsici led to a significant reduction of virulence in $N$. benthamiana and bell pepper, which could be restored by gene complementation. Overexpression of PcAvhl in P. capsici, on the other hand, resulted in increased virulence. PcAvh1 induced cell death when transiently expressed in $N$. benthamiana, tomato, and bell pepper. Subsequently, we characterized potential host targets of PcAvh1 and revealed that PcAvh1 interacts with the scaffolding subunit of the protein phosphatase 2A (PP2Aa). Virus-induced gene silencing (VIGS) of $P P 2 A a$ resulted in an increased susceptibility to $P$. capsici infection and dwarfism, indicating that PP2Aa plays a positive role in plant defense and growth. Overall, our data demonstrate that PcAvh1 is required for the full virulence of $P$. capsici, possibly by associating with PP2Aa.

\section{RESULTS}

\section{PcAvh1 is highly expressed during} in planta infection by $P$. capsici.

Our previous study identified a number of effectors associated with $P$. capsici pathogenicity, including PcAvh1 (GenBank accession number MF975713) (Chen et al. 2013). To further determine the virulence contribution of PcAvh1, we examined its expression profile at different developmental stages (mycelium, sporangium, zoospore, germinating cyst) and during infection $(1.5,3,6,12,24$, 36 , and $72 \mathrm{~h}$ postinoculation [hpi]) in $N$. benthamiana. We found that $P c A v h l$ had a minimal expression during $P$. capsici development without a host plant but was rapidly upregulated at the infection stages (Fig. 1). PcAvhl transcripts reached a maximal level at $1.5 \mathrm{hpi}$ and then declined onwards until $24 \mathrm{hpi}$, when its transcript was only weakly detected. At the later infection stages (36 and 72 hpi), $P c A v h l$ was upregulated again.

\section{PcAvh1 has allelic sequences in the $P$. capsici population} and homologs only in Phytophthora sister species.

We checked the presence of $P c A v h 1$ in 31 isolates (Supplementary Table S1) of $P$. capsici. High-fidelity PCR templates of both genomic DNA and 1.5-hpi cDNA from each isolate yielded the same products. Eleven alleles of $P c A v h 1$ were identified in all sequenced isolates (Fig. 2). Thirty-two site variations at the DNA level were detected in PcAvhl (Supplementary Fig. S1), leading to variations in 15 amino acids (Fig. 2 ). The strength of natural selection acting upon the effector was further examined by estimating the ratio of the nonsynonymous substitution rate $(\mathrm{dN})$ to the synonymous substitution rate (dS) (Table 1). We defined the $\mathrm{N}$ (including RXLR and dEER) and $C$ termini of the protein sequence on the basis of the cleaved site at the 47th amino acid. The Yn00 tool included in the PAML v4.3 software package (Yang 2007) was utilized to count the ratio of $\mathrm{dN}$ to $\mathrm{dS}$. The 11 sequences made up 55 pairwise comparisons for the test. The result showed that the $\mathrm{dN} / \mathrm{dS}$ values of the full-length sequence and the $\mathrm{C}$ terminus were lower than unity, suggesting evidence of

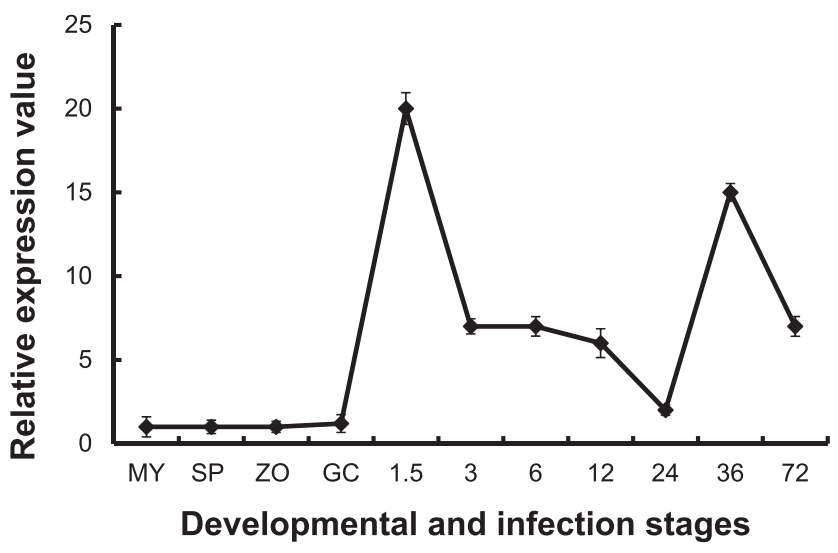

Fig. 1. Expression level of PcAvhl during the developmental and infection stages of Phytophthora capsici. MY = vegetative mycelia, $\mathrm{SP}=$ sporangia, $\mathrm{ZO}=$ zoospores, $\mathrm{GC}=$ germinating cysts; 1.5 to $72=N$. benthamiana roots at $1.5,3,6,12,24,36$, and $72 \mathrm{~h}$ postinoculation. Sterile water and cDNA from sterile water-inoculated plants were used as negative control templates in quantitative reverse transcription-PCR 
purifying selection pressure in the $P c A v h l$ gene, especially at the C-terminus of the sequence (Table 1). The maximumlikelihood analysis by the fixed effects likelihood (FEL) tool in the Datamonkey suite (Delport et al. 2010; Kosakovsky Pond and Frost 2005) revealed that PcAvh1 contains three residues $(276 \mathrm{D}, 305 \mathrm{D}, 331 \mathrm{~S})$ that are under negative selection $(P<0.05)$.

A BlastP search of the FungiDB, National Center for Biotechnology Information (NCBI), and genome databases of various oomycetes were further performed to determine the presence of PcAvh1 homologs in other organisms. Homologs were identified only in Phytophthora species, including P. infestans, P. sojae, $P$. ramorum, $P$. cinnamomi, $P$. parasitica, $P$. megakarya, $P$. cactorum, and $P$. palmivora, but not in fungi or other organisms. All of them contained the canonical signal peptide and RxLRdEER motif (Fig. 3A). The sequence alignment demonstrated a high conservation of PcAvh1 among different Phytophthora species (Fig. 3A). Phylogenetic analysis revealed the evolutionary relationship between PcAvh1 and its homologs, with the homolog (PHPALM28136) from $P$. palmivora as the closest relative (Fig. 3B). Both showed $76 \%$ similarity to each other (Fig. 3A). The homologs from $P$. infestans and $P$. parasitica form a clade distinct from others (Fig. 3B).

PcAvh1 is required for $P$. capsici infection of host plants.

Targeted gene replacement mediated by homology-directed repair (HDR) of double-strand break cut by single guide ( $\mathrm{sg}$ ) RNA-guided Cas9 has been proven to be an efficient gene KO method in P. sojae (Fang and Tyler 2016; Fang et al. 2017). To directly establish the virulence function of PcAvh1, we generated $\mathrm{KO}$ mutants in $P$. capsici using this method. sgRNAs targeting $P c A v h l$ were designed, using the eukaryotic pathogen CRISPR guide RNA/DNA design tool EuPaGDT, as suggested by Fang et al. (2017). The top-rated sgRNA candidates were further filtered by off-target analysis and secondary structure examination (Fang et al. 2017). Two sgRNAs targeting the Cterminus of PcAvh1 were selected (Supplementary Fig. S2). We cotransformed these two sgRNAs and the hSpCas9 expression plasmid together with the uncut donor DNA plasmid that contained the NPTII open reading frame flanked by approximately 1$\mathrm{kb}$ arm sequences surrounding the $P c A v h l$ gene. Subsequently, $N c o$ I digestion of gDNA PCR products and sequencing identified three independent PcAvhl KO lines (KO1 to KO3) in which $P c A v h 1$ transcripts were undetectable compared with the wildtype (WT) strain (Pc537) at 1.5 hpi (Fig. 4A). A non-HDR false positive transformation control expressing $P c A v h l$ at a level similar to that in WT (Fig. 4A) and showing unaltered pathogenicity (Fig. 4B and C) served as a negative control. All transformants showed similar filamentous growth and zoospore production with WT. To assess their virulence, zoospores were inoculated onto the leaves of $N$. benthamiana (Fig. 4D and E) and the stems of bell pepper seedlings (Fig. 4F and G). KO of $P c A v h l$ significantly impaired the virulence of $P$. capsici (Fig. 4D to $\mathrm{G})$.

We next generated three complementation strains ( $\mathrm{C} 1$ to $\mathrm{C} 3$ ) by introducing pTORmRFP4 (Torche 2004) harboring the fulllength $P c A v h l$ into the $P c A v h 1 \mathrm{KO}$ mutants. Expression of $P c A v h l$ was restored in these complementation strains to levels comparable to WT (Fig. 4A); consequently, the reduced virulence activity of these $\mathrm{KO}$ mutants was also restored (Fig. $4 \mathrm{H}$ and I). To further elucidate the virulence function of PcAvh1,

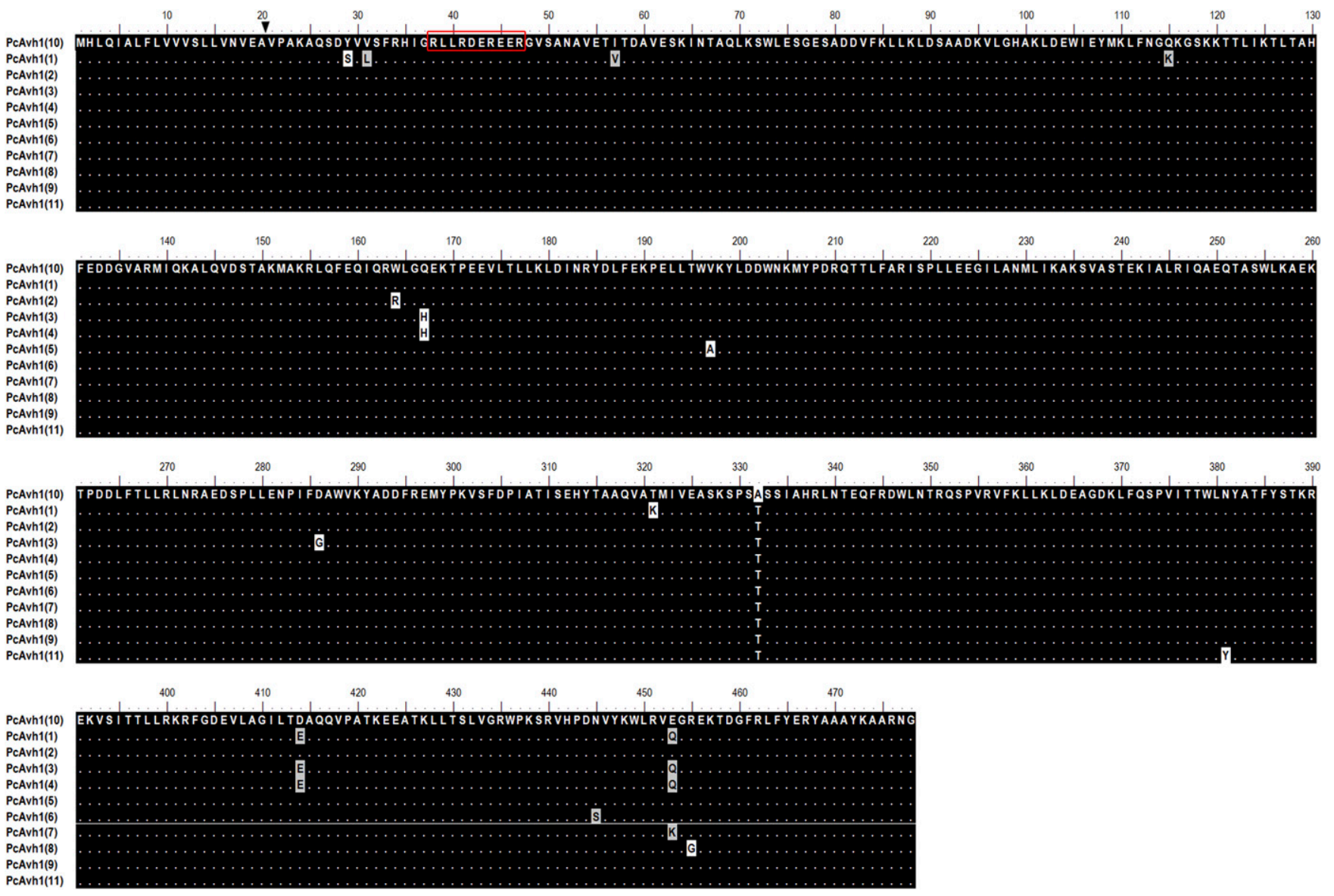

Fig. 2. Sequence comparison of $P c A v h 1$ alleles at the amino acid level. The sequence from the isolate Pc537 was on the top, labeled as PcAvh1(10). Identities are indicated by dots and substitutions by the substituted residues. The canonical RxLR-dEER motif is boxed and the cleavage site of signal peptide is indicated by an arrow on the top of the sequences. 
we generated two overexpression strains (OX1 and OX2) by introducing pTORmRFP4::PcAvhl into the WT strain (Fig. 4A). In contrast to the KO mutants, the OX1 and OX2 strains showed increased virulence compared with WT (Fig. 4J and K). All the strains, including the $\mathrm{KO}$ mutants, the complementation strains, and the overexpression strains are similar to WT and the control in mycelial growth and zoospore formation. These results together suggest that PcAvh1, although dispensable for development, is required for the full virulence of $P$. capsici.
Ectopic expression of PcAvh1 triggers cell death in $N$. benthamiana, tomato, and pepper leaves and compensates the virulence defect of the KO mutants.

Agrobacterium tumefaciens-mediated transformation (agroinfection) was utilized to transiently express $P c A v h l$ in the leaves of $N$. benthamiana, tomato (cultivars L402, Zhongza-9, Maofen-802) and bell pepper (Fig. 5). The coding sequence of PcAvh1 excluding the N-terminal secretion signal was cloned into the binary Potato virus $X$ vector pGR107 (Jones et al.

Table 1. Nonsynonymous substitution to synonymous substitution (dN/dS) analysis of pairwise comparisons of $P c A v h 1$ alleles

\begin{tabular}{|c|c|c|c|c|c|c|c|c|c|c|}
\hline \multicolumn{2}{|c|}{$\begin{array}{l}\text { Pairwise } \\
\text { comparison }\end{array}$} & \multicolumn{3}{|c|}{ Full sequence } & \multicolumn{3}{|c|}{ N-terminus ${ }^{a}$} & \multicolumn{3}{|c|}{ C-terminus ${ }^{a}$} \\
\hline Seq. 1 & $\overline{\text { Seq. } 2}$ & $d N / d S^{b}$ & $\mathbf{d N}$ & dS & $\mathbf{d N} / \mathbf{d} \mathbf{S}^{\mathbf{b}}$ & $d \mathbf{N}$ & dS & $\mathbf{d N} / \mathbf{d S}^{\mathbf{b}}$ & dN & dS \\
\hline 2 & 1 & 0.2083 & 0.0010 & 0.0048 & $\infty$ & 0.0236 & 0 & 0.2037 & 0.0011 & 0.0054 \\
\hline 3 & 1 & 0.2083 & 0.0010 & 0.0048 & - & 0 & 0 & 0.2037 & 0.0011 & 0.0054 \\
\hline 3 & 2 & $\infty$ & 0.0020 & 0 & $\infty$ & 0.0236 & 0 & $\infty$ & 0.0022 & 0 \\
\hline 4 & 1 & 0.2083 & 0.0010 & 0.0048 & - & 0 & 0 & 0.2037 & 0.0011 & 0.0054 \\
\hline 4 & 2 & $\infty$ & 0.0020 & 0 & $\infty$ & 0.0236 & 0 & $\infty$ & 0.0022 & 0 \\
\hline 4 & 3 & $\infty$ & 0.0020 & 0 & - & 0 & 0 & $\infty$ & 0.0022 & 0 \\
\hline 5 & 1 & 0.2083 & 0.0010 & 0.0048 & - & 0 & 0 & 0.2037 & 0.0011 & 0.0054 \\
\hline 5 & 2 & $\infty$ & 0.0020 & 0 & $\infty$ & 0.0236 & 0 & $\infty$ & 0.0022 & 0 \\
\hline 5 & 3 & $\infty$ & 0.0020 & 0 & - & 0 & 0 & $\infty$ & 0.0022 & 0 \\
\hline 5 & 4 & $\infty$ & 0.0020 & 0 & - & 0 & 0 & $\infty$ & 0.0022 & 0 \\
\hline 6 & 1 & 0.2083 & 0.0010 & 0.0048 & - & 0 & 0 & 0.2037 & 0.0011 & 0.0054 \\
\hline 6 & 2 & $\infty$ & 0.0020 & 0 & $\infty$ & 0.0236 & 0 & $\infty$ & 0.0022 & 0 \\
\hline 6 & 3 & $\infty$ & 0.0020 & 0 & - & 0 & 0 & $\infty$ & 0.0022 & 0 \\
\hline 6 & 4 & $\infty$ & 0.0020 & 0 & - & 0 & 0 & $\infty$ & 0.0022 & 0 \\
\hline 6 & 5 & $\infty$ & 0.0020 & 0 & - & 0 & 0 & $\infty$ & 0.0022 & 0 \\
\hline 7 & 1 & 0.2083 & 0.0010 & 0.0048 & - & 0 & 0 & 0.2037 & 0.0011 & 0.0054 \\
\hline 7 & 2 & $\infty$ & 0.0020 & 0 & $\infty$ & 0.0236 & 0 & $\infty$ & 0.0022 & 0 \\
\hline 7 & 3 & $\infty$ & 0.0020 & 0 & - & 0 & 0 & $\infty$ & 0.0022 & 0 \\
\hline 7 & 4 & $\infty$ & 0.0020 & 0 & - & 0 & 0 & $\infty$ & 0.0022 & 0 \\
\hline 7 & 5 & $\infty$ & 0.0020 & 0 & - & 0 & 0 & $\infty$ & 0.0022 & 0 \\
\hline 7 & 6 & $\infty$ & 0.0020 & 0 & - & 0 & 0 & $\infty$ & 0.0022 & 0 \\
\hline 8 & 1 & 0.2083 & 0.0010 & 0.0048 & - & 0 & 0 & 0.0919 & 0.0033 & 0.0359 \\
\hline 8 & 2 & $\infty$ & 0.0020 & 0 & $\infty$ & 0.0236 & 0 & 0.1457 & 0.0044 & 0.0302 \\
\hline 8 & 3 & $\infty$ & 0.0020 & 0 & - & 0 & 0 & 0.1457 & 0.0044 & 0.0302 \\
\hline 8 & 4 & $\infty$ & 0.0020 & 0 & - & 0 & 0 & 0.1457 & 0.0044 & 0.0302 \\
\hline 8 & 5 & $\infty$ & 0.0020 & 0 & - & 0 & 0 & 0.1093 & 0.0033 & 0.0302 \\
\hline 8 & 6 & $\infty$ & 0.0020 & 0 & - & 0 & 0 & 0.1457 & 0.0044 & 0.0302 \\
\hline 8 & 7 & $\infty$ & 0.0020 & 0 & - & 0 & 0 & 0.1457 & 0.0044 & 0.0302 \\
\hline 9 & 1 & 0.1130 & 0.0039 & 0.0345 & - & 0 & 0 & 0.1134 & 0.0044 & 0.0388 \\
\hline 9 & 2 & 0.1661 & 0.0049 & 0.0295 & $\infty$ & 0.0236 & 0 & 0.1667 & 0.0055 & 0.0330 \\
\hline 9 & 3 & 0.1667 & 0.0049 & 0.0294 & - & 0 & 0 & 0.1667 & 0.0055 & 0.0330 \\
\hline 9 & 4 & 0.1667 & 0.0049 & 0.0294 & - & 0 & 0 & 0.1667 & 0.0055 & 0.0330 \\
\hline 9 & 5 & 0.1667 & 0.0049 & 0.0294 & - & 0 & 0 & 0.1333 & 0.0044 & 0.0330 \\
\hline 9 & 6 & 0.1667 & 0.0049 & 0.0294 & - & 0 & 0 & 0.1631 & 0.0054 & 0.0331 \\
\hline 9 & 7 & 0.1661 & 0.0049 & 0.0295 & - & 0 & 0 & 0.1667 & 0.0055 & 0.0330 \\
\hline 9 & 8 & 0.1322 & 0.0039 & 0.0295 & - & 0 & 0 & 0.4074 & 0.0011 & 0.0027 \\
\hline 10 & 1 & 0.0938 & 0.0030 & 0.0320 & - & 0 & 0 & 0.1964 & 0.0054 & 0.0275 \\
\hline 10 & 2 & 0.1444 & 0.0039 & 0.0270 & $\infty$ & 0.0236 & 0 & 0.2968 & 0.0065 & 0.0219 \\
\hline 10 & 3 & 0.1449 & 0.0039 & 0.0269 & - & 0 & 0 & 0.2968 & 0.0065 & 0.0219 \\
\hline 10 & 4 & 0.1449 & 0.0039 & 0.0269 & - & 0 & 0 & 0.2968 & 0.0065 & 0.0219 \\
\hline 10 & 5 & 0.1449 & 0.0039 & 0.0269 & - & 0 & 0 & 0.2466 & 0.0054 & 0.0219 \\
\hline 10 & 6 & 0.1449 & 0.0039 & 0.0269 & - & 0 & 0 & 0.2968 & 0.0065 & 0.0219 \\
\hline 10 & 7 & 0.1444 & 0.0039 & 0.0270 & - & 0 & 0 & 0.2968 & 0.0065 & 0.0219 \\
\hline 10 & 8 & 0.1111 & 0.0030 & 0.0270 & - & 0 & 0 & 0.1781 & 0.0044 & 0.0247 \\
\hline 10 & 9 & 0.4167 & 0.0010 & 0.0024 & - & 0 & 0 & 0.1964 & 0.0054 & 0.0275 \\
\hline 11 & 1 & 0.2816 & 0.0069 & 0.0245 & - & 0 & 0 & 0.2037 & 0.0011 & 0.0054 \\
\hline 11 & 2 & 0.4051 & 0.0079 & 0.0195 & $\infty$ & 0.0236 & 0 & $\infty$ & 0.0022 & 0 \\
\hline 11 & 3 & 0.4051 & 0.0079 & 0.0195 & - & 0 & 0 & $\infty$ & 0.0022 & 0 \\
\hline 11 & 4 & 0.4051 & 0.0079 & 0.0195 & - & 0 & 0 & $\infty$ & 0.0022 & 0 \\
\hline 11 & 5 & 0.4051 & 0.0079 & 0.0195 & - & 0 & 0 & $\infty$ & 0.0022 & 0 \\
\hline 11 & 6 & 0.4051 & 0.0079 & 0.0195 & - & 0 & 0 & $\infty$ & 0.0022 & 0 \\
\hline 11 & 7 & 0.4051 & 0.0079 & 0.0195 & - & 0 & 0 & $\infty$ & 0.0022 & 0 \\
\hline 11 & 8 & 0.3538 & 0.0069 & 0.0195 & - & 0 & 0 & 0.1457 & 0.0044 & 0.0302 \\
\hline 11 & 9 & 0.2816 & 0.0069 & 0.0245 & - & 0 & 0 & 0.1667 & 0.0055 & 0.0330 \\
\hline 11 & 10 & 0.2682 & 0.0059 & 0.0220 & - & 0 & 0 & 0.2968 & 0.0065 & 0.0219 \\
\hline
\end{tabular}

\footnotetext{
${ }^{a}$ The N-terminus refers to the sequence from the first to the end of dEER, and the C-terminus refers to the sequence from the immediate residue after dEER to the end, except the stop codon.

$\mathrm{b} \infty$ indicates pairwise comparisons in which $\mathrm{dN}>0$ and $\mathrm{dS}=0$, and - refers to pairwise comparisons in which $\mathrm{dN}=0$ and $\mathrm{dS}=0$.
} 
1999). Transient expression of PcAvhl induced HR-like cell death as vigorously as the positive controls INF1 (Bos et al. 2006) and SCR99 (Chen et al. 2016) in N. benthamiana (Fig. 5A) and tomato (Fig. 5B), respectively. We also checked the phenotype when $P c A v h l$ was expressed in bell pepper, one of the natural hosts of the pathogen, and observed mild cell-death symptoms in the leaves (Fig. 5C). To further confirm the HRtriggering activity, we cloned $P c A v h 1$, again without the signal peptide, into another binary vector pEarleyGate100 (Earley et al. 2006). Similarly, expression of PcAvhl driven by the
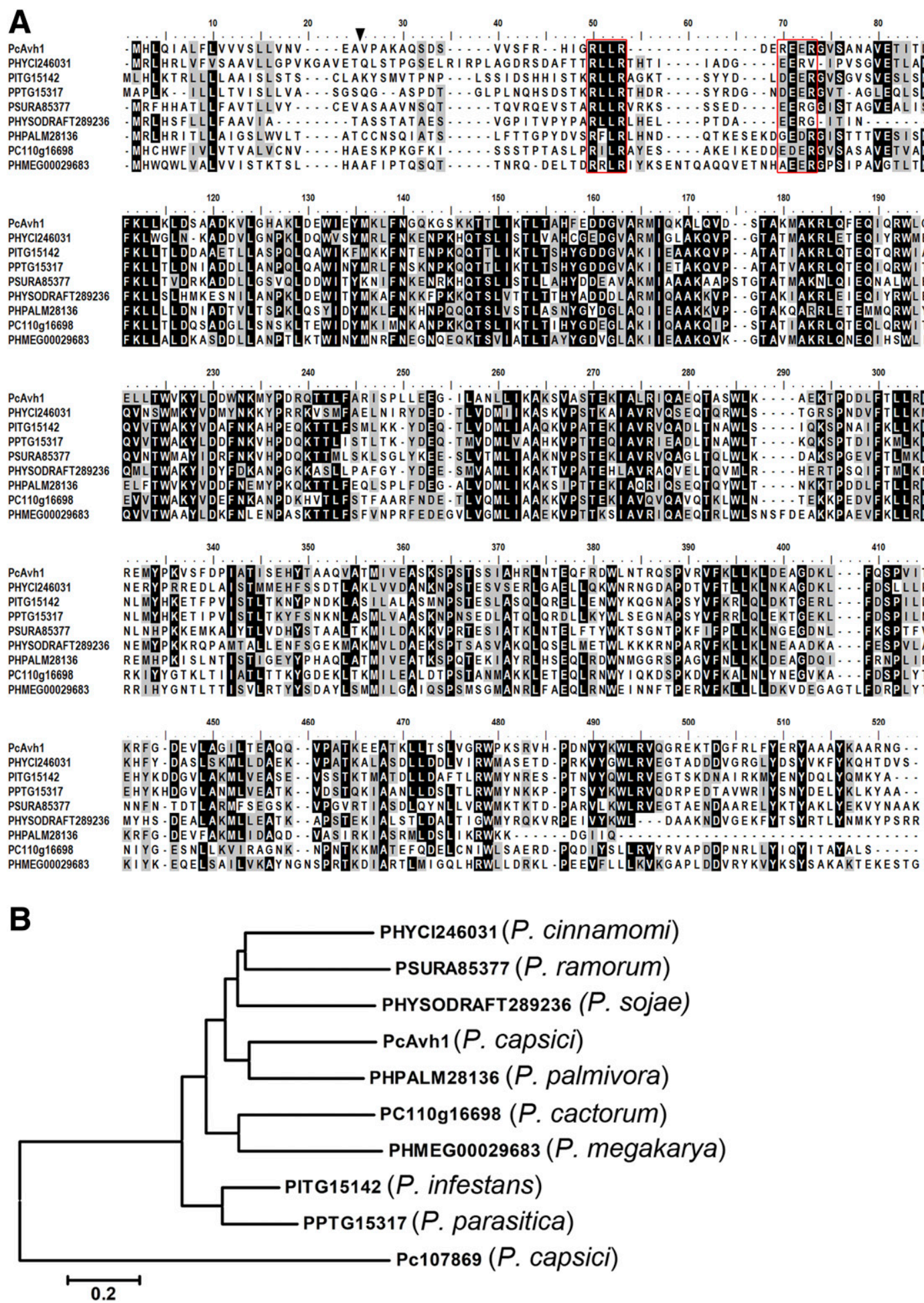

Fig. 3. Protein sequence alignment and phylogenetic analysis of Phytophthora capsici PcAvh1 and its homologs in different Phytophthora species. A, Protein sequences were aligned and shaded for consensus (60\% threshold for shading). The characteristic motifs RxLR and dEER are boxed. The predicted cleavage site for the signal peptide of PcAvh1 is indicated by an arrow on the top of the sequences. B, Phylogeny of PcAvh1 and homologs with locus tags from different Phytophthora species. Five homologs (PITG_15142, PHYSODRAFT_289236, PSURA_85377, PHYCI_246031, and PPTG_15317) of PcAvh1 were downloaded from FungiDB, while the other three (PHPALM_28136, PC110g16698, and PHMEG_00029683) from the National Center for Biotechnology Information database. The P. capsici NLP effector Pc107869 (Chen et al. 2018) served as an outgroup in the neighbor-joining tree. 
cauliflower mosaic virus $35 S$ promoter also caused cell death in $N$. benthamiana at 5 days postinfiltration (dpi) (Fig. 5D).

To determine if the cell death-triggering activity of PcAvh1 is associated with its virulence role, the $P$. capsici mutant $\mathrm{KO} 3$ was inoculated onto the $N$. benthamiana plants expressing $P c A v h 1$ with pEarleyGate 100 at 2 dpi, when cell death induced by PcAvh1 is not yet visible. As stated above, KO of PcAvh1 significantly impaired the virulence of $P$. capsici on
$N$. benthamiana leaves agroinfiltrated with empty pEarleyGate100 (Fig. 6A). In contrast, we observed that prior overexpression of PcAvhl in N. benthamiana compensates the virulence defect of the mutant KO3 (Fig. 6A and B). The same phenomenon was observed for $\mathrm{KO} 1$ and $\mathrm{KO} 2$ on the plants expressing PcAvhl (only KO3 was shown here as the representative). The infection of the CRISPR/Cas9 transformation control was also promoted by the transient
A

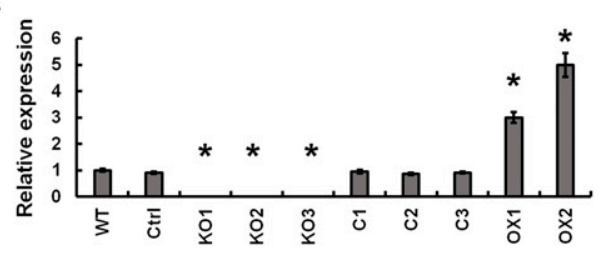

B

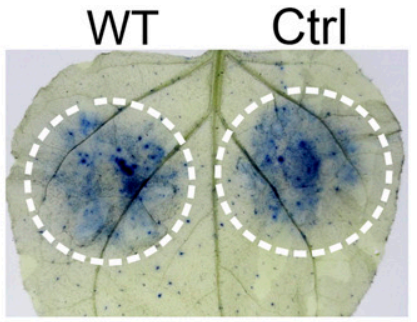

C

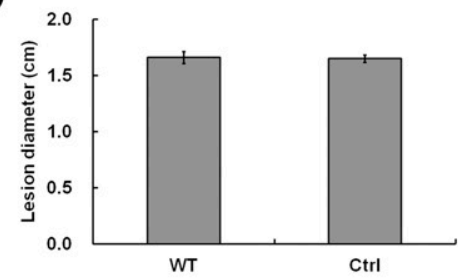

E
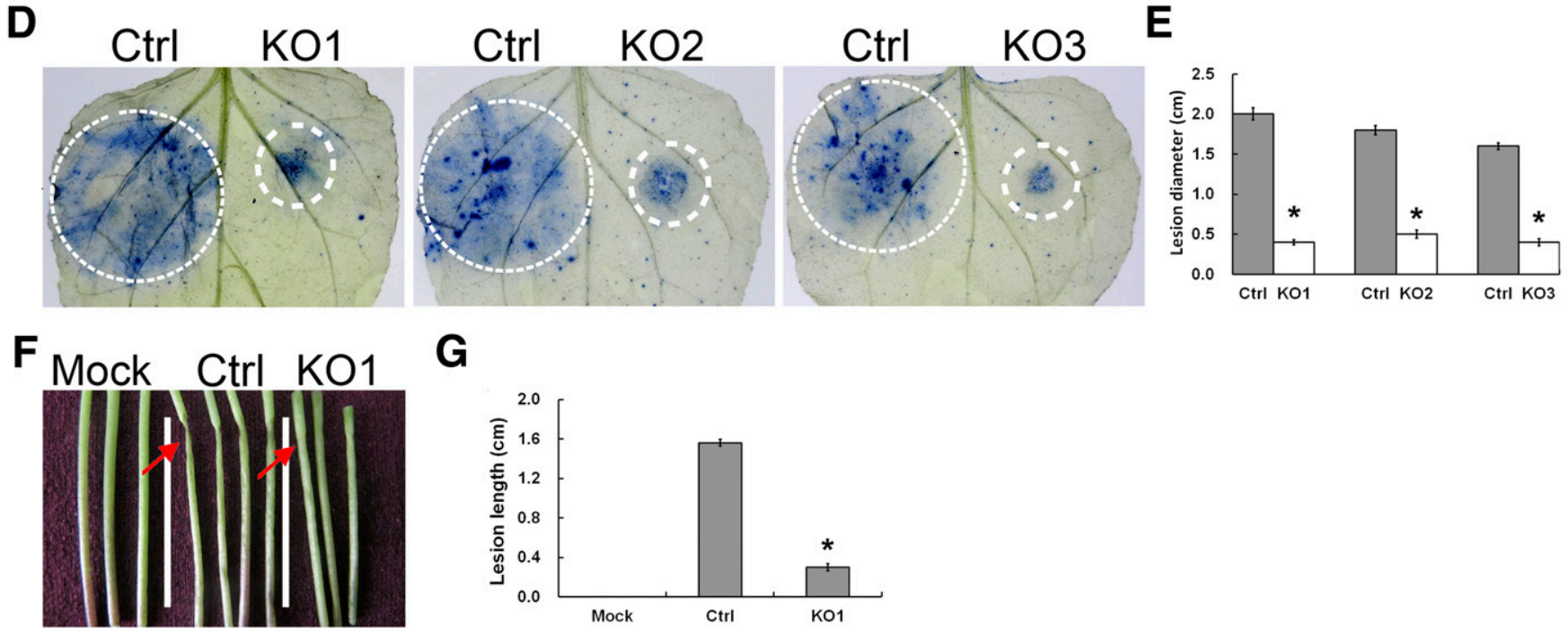

G

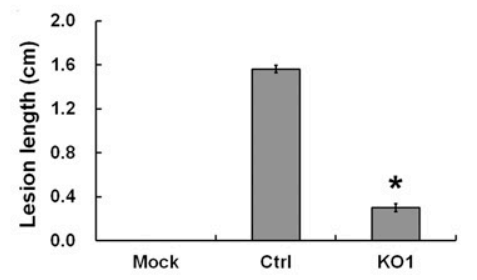

H
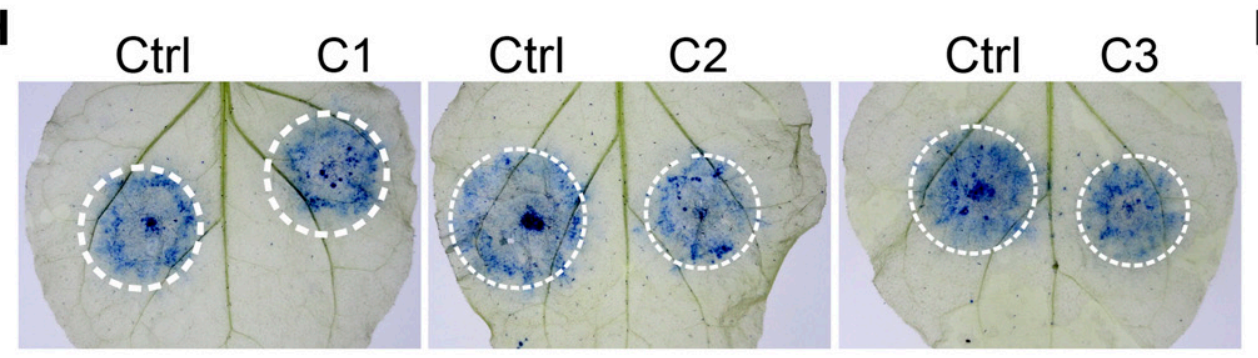

I
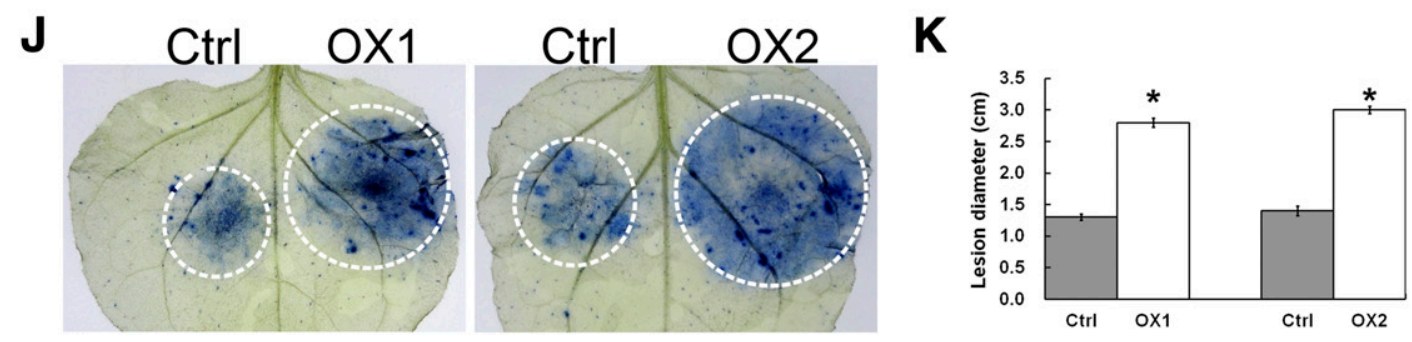

Fig. 4. PcAvh1 is an important virulence factor of Phytophthora capsici. A, The transcript abundances of $P c A v h 1$ were determined at $1.5 \mathrm{~h}$ postinoculation (hpi) in three CRISPR/Cas9-mediated gene knockout (KO) transformants (KO1 to KO3), three KO gene-complemented transformants (C1 to C3), two geneoverexpression transformants (OX1, OX2), a non-homology-directed repair false positive transformation control (Ctrl), and the wild-type (WT) strain Pc537. B to K, Virulence activities of the $P$. capsici strains in Nicotiana benthamiana (B to E, H to K) and bell pepper (F and G). The graphs in C, E, G, I, and K show results of statistical analysis of lesion sizes in the images shown in B, D, F, H, and J. Error bars are standard error and the asterisks $(*)$ denote statistically significant difference (Student $t$ test, $P<0.01$ ). Mock means sterile water inoculation and arrows in F indicate the lesions. Photographs were taken at 48 (B and D) or $24(\mathrm{~F}, \mathrm{H}$, and $\mathrm{G})$ hpi. 

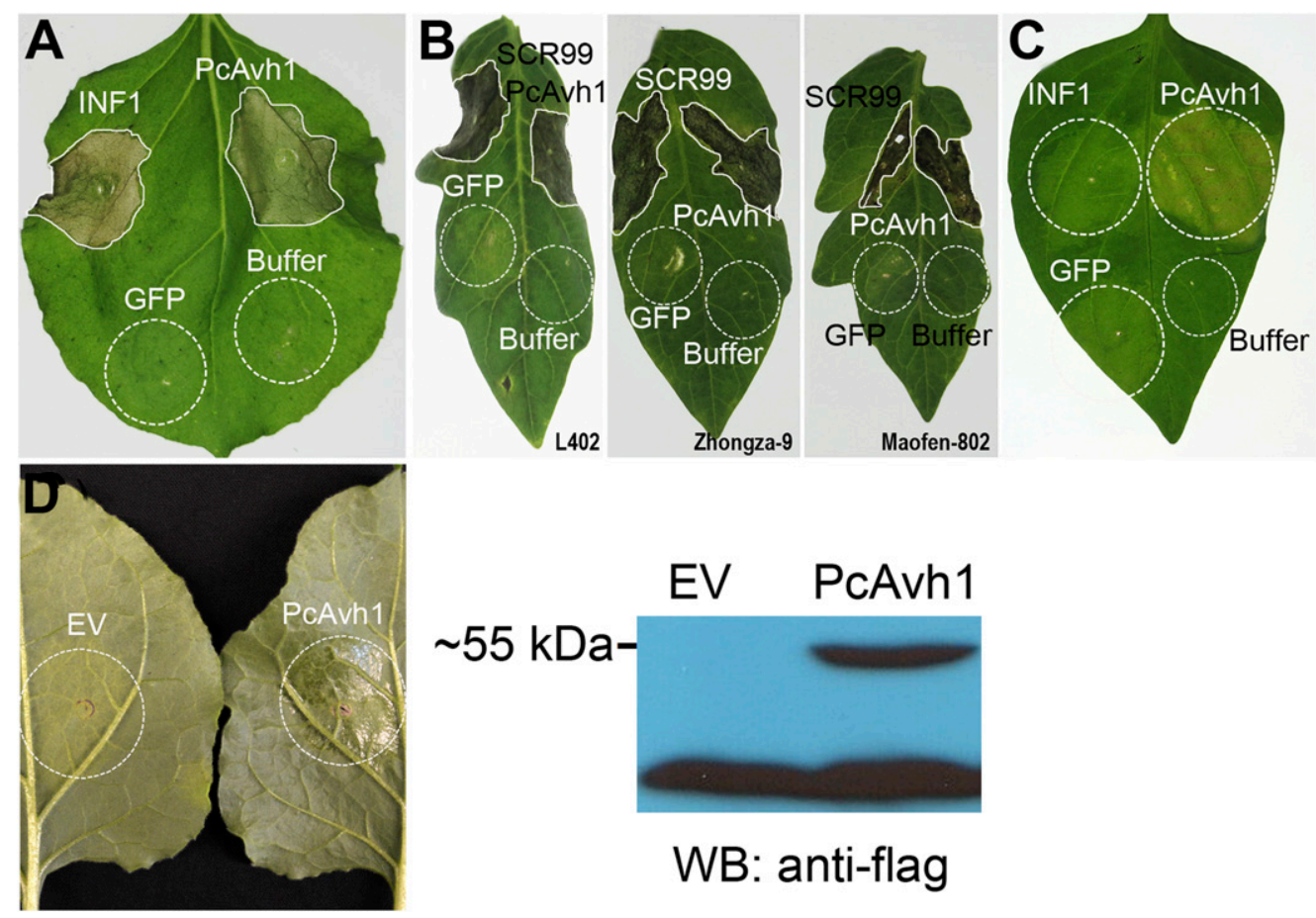

\section{WB: anti-flag}

Fig. 5. Transient expression of $P c A v h 1$ in family Solanaceae plants triggers cell death. A to C, Leaves of Nicotiana benthamiana, tomato, and bell pepper, respectively, were infiltrated with Agrobacterium tumefaciens GV3101 cells harboring pGR107-PcAvh1 and D, leaves of $N$. benthamiana infiltrated with GV3101 cells harboring pEarleyGate100-PcAvh1. Phytophthora infestans elicitin INF1 served as a positive control in N. benthamiana (A), while Phytophthora cactorum SCR99 was used as a positive control in tomato (B). Green fluorescent protein (GFP) and $10 \mathrm{mM} \mathrm{MgCl}_{2}$ buffer were used as negative controls in all tested plants except in D, where empty pEarleyGate100 vector (EV) served as the negative control. Protein expression, e.g., in D, was validated by Western blotting using antiflag antibody. The circles indicate the infiltration areas. Photographs were taken 5 (A and D) or 7 (B and C) days after infiltration.

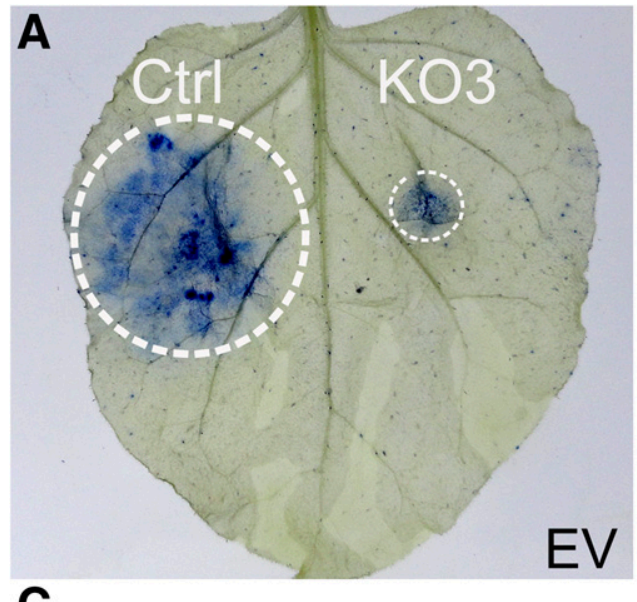

C

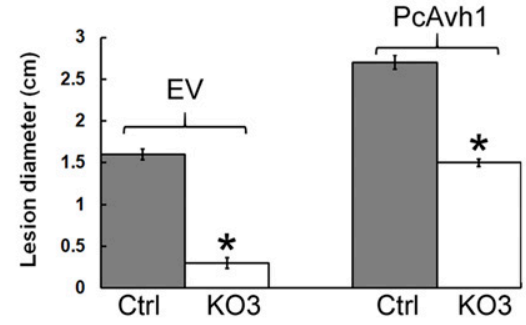

B

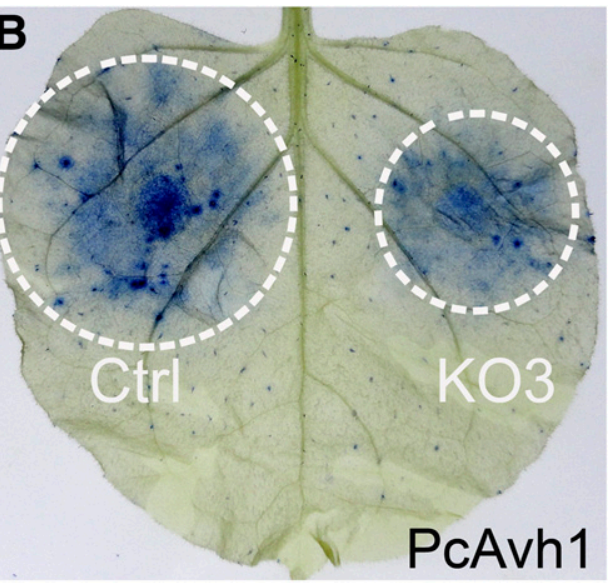

D

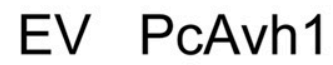

$\sim 55 \mathrm{kDa}$

Fig. 6. Overexpression of $P c A v h 1$ in Nicotiana benthamiana compensates the virulence defect of the PcAvh1 knockout. A, The leaves of $N$. benthamiana infiltrated with GV3101 cells harboring empty pEarleyGate100 vector (EV) or B, pEarleyGate100-PcAvh1. Ctrl = a non-homology-directed repair false positive transformation control, KO3 = a CRISPR/Cas9-mediated PcAvhl knockout transformant. C, Statistical analysis of lesion size in A and B. Error bars are standard error and the asterisks (*) denote statistically significant difference (Student $t$ test, $P<0.01$ ). D, The expression of PcAvh1 in leaves was validated by Western blotting using antiflag antibody. The circles indicate the infiltration areas. Photographs were taken 2 days after infiltration. 
expression of $P c A v h l$ (Fig. 6A to C). Altogether, these results indicate that the cell death-triggering activity of PcAvh1 is associated with its virulence activity.

PcAvh1 interacts with plant PP2A A subunit.

To understand the mechanism underlying the virulence activity of PcAvh1, we investigated its potential host target (or targets) using yeast two-hybrid (Y2H) screening. A cDNA library was created using RNAs extracted from $N$. benthamiana infected with the $P$. capsici isolate Pc537 at 15 hpi (during the biotrophic stage) and 72 hpi (during the necrotrophic stage). The library was screened to a depth of $1.6 \times 10^{6}$ yeast cotransformants with a GAL4 DNA-binding domain-PcAvh1 fusion construct. In total, 12 yeast colonies expressing potential
A

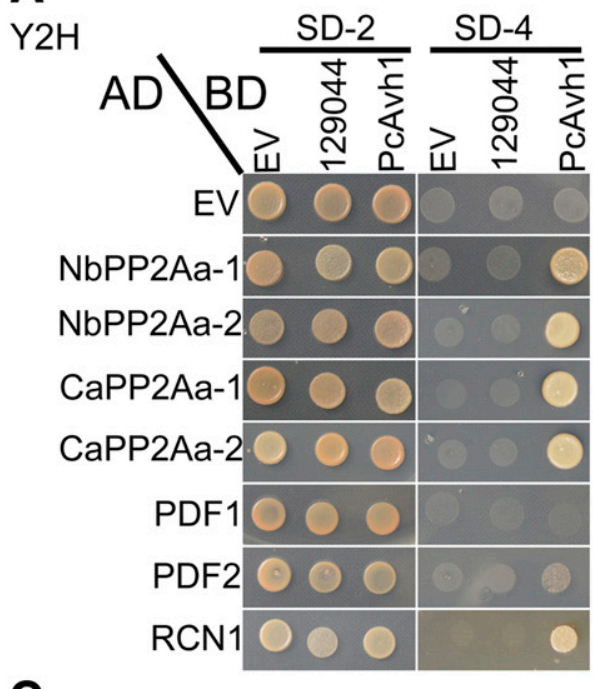

C
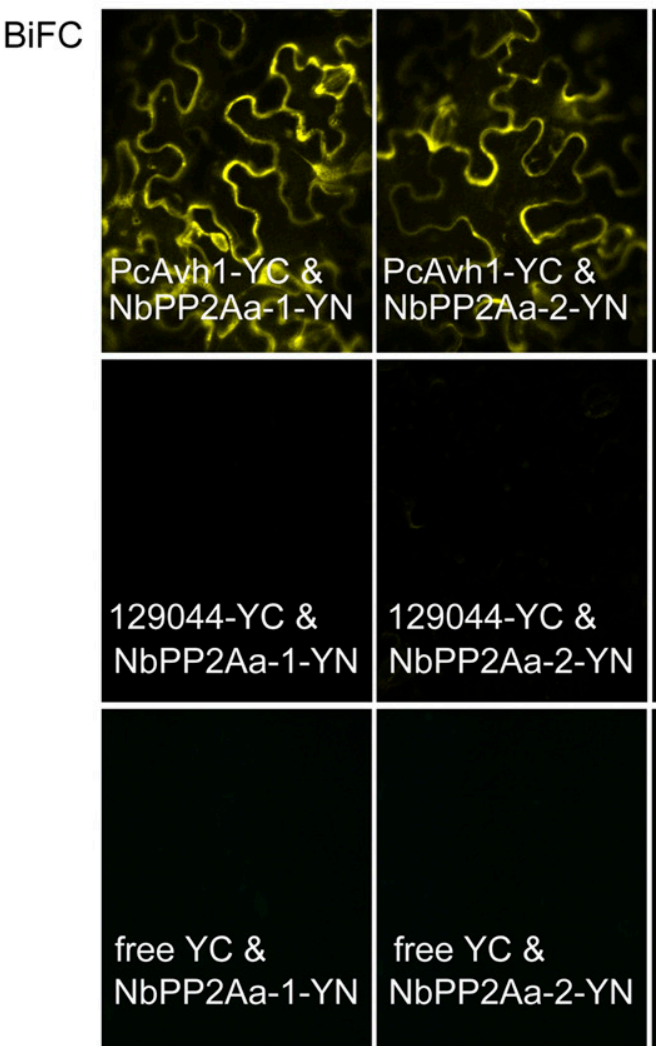

B

GST pulldown

$\frac{\text { PcAvh1 }}{-N-N_{N}} \frac{129044}{-N^{2}-N_{N}} \frac{\text { pRSF }}{-T-N}$

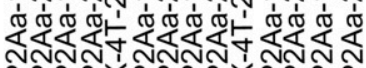

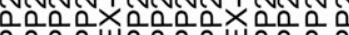

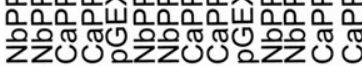
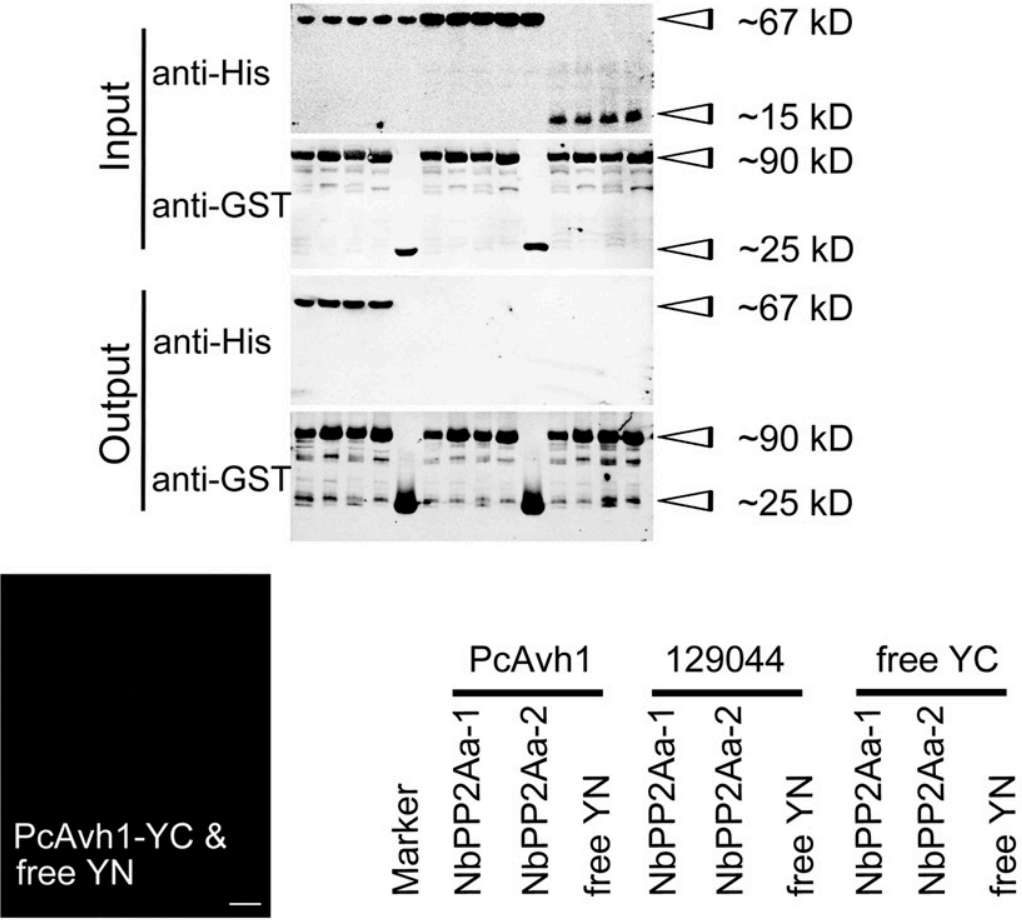

$75 \mathrm{kD}$ $\sim 63 \mathrm{kD}$

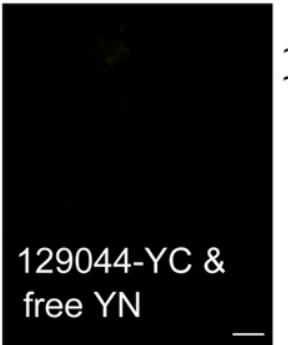

Fig. 7. PcAvh1 interacts with two isoforms of PP2Aa subunit. A, PcAvh1 interacts with two isoforms of PP2Aa of both Nicotiana benthamiana (NbPP2Aa-1 and NbPP2Aa-2) and bell pepper (CaPP2Aa-1 and CaPP2Aa-2) in yeast, one isoform of PP2Aa in Arabidopsis (RCN1). Another P. capsici RXLR effector, 129044, was used as a negative control. Yeast transformants were grown on nonselective SD/-Trp/-Leu (SD-2) medium or the selective medium SD/Trp/-Leu/-His/-Ade (SD-4). Plates were photographed at 5 days after inoculation. EV = empty BD (pGBKT7) or AD (pGADT7) vector. B, In vitro pull-down of His-PcAvh1 with GST-NbPP2Aa-1, GST-NbPP2Aa-2, GST-CaPP2Aa-1, and GST-CaPP2Aa-2. His-PcAvh1, His-129044, and GST-PP2Aa were expressed in Escherichia coli. Coprecipitation of PP2Aa with effectors was examined by Western blotting. pRSF = the empty vector pRSFDuet-1. C, Confocal microscopy of $N$. benthamiana leaves coexpressing the C-terminal portion (YC)-PcAvh1 with the N terminus (YN)-NbPP2Aa-1 or YN-NbPP2Aa-2. Controls include leaves expressing YC-129044 with either YN-NbPP2Aa-1 or YN-NbPP2Aa-2, YC with either YN-NbPP2Aa-1 or YN-NbPP2Aa-2, YC-PcAvh1 with YN, YC-129044 with YN, and YC with YN. Bars $=20 \mu \mathrm{m}$. 
PcAvh1-interacting proteins were recovered. Sequences from nine of the 12 clones encode an isoform of PP2A scaffolding subunit (PP2Aa), while the remaining three clones correspond to another isoform of the same protein, hereafter called NbPP2Aa-1 (MF996339) and NbPP2Aa-2 (MF996340), respectively. These two isoforms share $90 \%$ identity in amino acid sequences. Supplementary Fig. S3 shows an amino acid alignment of NbPP2Aa proteins with the homologous subunits from Arabidopsis (PDF1, PDF2, and RCN1), Oryza sativa (XP_015612104, XP_015612105), N. tabacum (CAA66487), and bell pepper (XM_016692206 and XM_016700428, designated as CaPP2Aa-1 and CaPP2Aa-2, respectively). To confirm the interaction, pairwise $\mathrm{Y} 2 \mathrm{H}$ was conducted with the prey clones of full-length NbPP2Aa-1 and NbPP2Aa-2 as well as CaPP2Aa-1 and CaPP2Aa-2 (Fig. 7A). Another RXLR effector, 129044, structurally similar to PcAvh1 (Ye and Ma 2016), was used as a negative control. The results show that only PcAvh1 interacts with NbPP2Aa proteins (Fig. 7A). PcAvh1 also interacts with the two CaPP2Aa proteins (Fig. 7A), indicating that PcAvh1 associates with a common protein in these two plants. Noticeably, PcAvh1 interacts with Arabidopsis RCN1 (Fig. 7A).

To further validate the interaction, an in-vitro pull-down assay was performed using glutathione $S$-transferase (GST)tagged PP2Aa (both from $N$. benthamiana and bell pepper) and His-tagged effectors (PcAvh1 and 129044) that were expressed in Escherichia coli. GST-tagged PP2Aa was immobilized on GST-resin and the enrichment of His-tagged effectors on the resin was examined. As shown in Figure 7B, only His-PcAvh1 protein was specifically enriched in GST-NbPP2Aa-1-bound, GST-NbPP2Aa-2-bound, GST-CaPP2Aa-1-bound, or GSTCaPP2Aa-2-bound glutathione resins, suggesting that PcAvh1 directly interacts with NbPP2Aa or CaPP2Aa in vitro.

We next examined the interaction of PcAvh1 with NbPP2Aa in planta using the bimolecular fluorescence complementation (BiFC) assay. The C-terminal portion (YC) of yellow fluorescent protein (YFP) was fused to PcAvh1 or 129044, while the N terminus (YN) was fused to NbPP2Aa. Western blot showed that all proteins were expressed well (Fig. 7C). Coexpression of YC-PcAvh1 with either YN-NbPP2Aa-1 or YN-NbPP2Aa-2 in $N$. benthamiana generated strong fluorescence signal in the nucleus and the cytoplasm, whereas no fluorescence was detected in cells coexpressing YC-129044 and either YNNbPP2Aa-1 or YN-NbPP2Aa-2 or in cells expressing, alone, YC-PcAvh1, YC-129044, or YN-NbPP2Aa (Fig. 7C). This result confirmed the existence of the interaction of PcAvh1 with NbPP2Aa. We also examined the subcellular localization of PcAvh1 in plant cells. The coding sequence of PcAvh1, excluding the secretion signal, was cloned into pBinGFP4 (Liu et al. 2014) to create the C-terminal fusion with green fluorescent protein (GFP). Localization of PcAvh1-GFP was examined in $N$. benthamiana leaf pavement cells (Supplementary Fig. S4). We found that PcAvh1 proteins were localized in the nucleus and the cytoplasm. To confirm the localizations, we coexpressed PcAvh1-GFP with the Arabidopsis plasma membrane aquaporin PIP2A (Cutler et al. 2000) fused with mCherry. Distinct with the plasma membrane localization of AtPIP2A-mCherry, PcAvh1-GFP was localized to nucleus and cytoplasm, which is in accordance with the BiFC result.

\section{Silencing of NbPP2Aa attenuates plant growth and resistance to $P$. capsici.}

To establish the potential role of PP2Aa in plant defense against $P$. capsici infection, we knocked down the expression of $N b P P 2 A a$ genes in $N$. benthamiana using VIGS (Fig. 8). Since the two NbPP2Aa isoforms are highly conserved, the divergent segments at the $5^{\prime}$ and $3^{\prime}$ untranslated regions from each gene were cloned into pBinary tobacco rattle virus (TRV) vector (pTRV2) in order to silence one gene at a time (Supplementary Fig. S5). The transcript abundance of NbPP2Aa-1 and NbPP2Aa-2 was decreased by 85 to $95 \%$ in plants expressing the isoform-specific construct (Supplementary Fig. S6). No defects of plant growth and development were observed in these $P P 2 A a$-silenced plants (Fig. 8A and D). However, silencing of either $N b P P 2 A a-1$ or $N b P P 2 A a-2$ resulted in increased susceptibility, reflected by the significantly enlarged lesions formed by $P$. capsici infection (Student $t$ test, $P<0.01$ ) (Fig. 8A and B). Furthermore, a 573-bp coding segment shared by the two isoform genes was cloned into pTRV2 (pTRV2$P P 2 A$ ) to silence both $N b P P 2 A a$ genes simultaneously. Similarly, these plants showed a significantly impaired resistance against the infection of $P$. capsici (Fig. 8A and B). Surprisingly, silencing of both $N b P P 2 A a$ genes caused dwarfism, suggesting that these two isoforms may function in redundancy in regulating plant development (Fig. 8A, C, and D). Intriguingly, transient expression of $P c A v h l$ still induces cell death in either single- or double-gene silenced plants (Fig. 8E), suggesting that PP2Aa is not required for PcAvh1-triggered cell death. We further inoculated the PcAvhl KO strains onto $P P 2 A a$ double-gene silenced plants and observed that silencing of $P P 2 A a$ genes in $N$. benthamiana compensates partially the virulence defect of the PcAvhl KO strains (Fig. 8F, KO3 as an example).

\section{DISCUSSION}

Plant pathogens employ a diverse array of secreted effector proteins to target a variety of host cell processes in order to facilitate the establishment of parasitic infection (Franceschetti et al. 2017; Kamoun 2006). Characterization of effector functions and their host targets is a critical step toward a better understanding of pathogenesis and plant immunity. Here, we demonstrate that the $P$. capsici RXLR effector PcAvh1, which is only expressed during infection, triggers cell death when expressed in $N$. benthamiana, tomato, and bell pepper leaves (Figs. 1 and 5). CRISPR/Cas9 KO of PcAvhl significantly impairs the virulence of $P$. capsici, while its stable overexpression increases the virulence (Fig. 4). Furthermore, ectopic expression of PcAvh1 in N. benthamiana compensates the virulence defect of the $\mathrm{KO}$ mutants (Fig. 6). Interestingly, PcAvh1 interacts with two host PP2A scaffolding isoforms (PP2Aa-1 and PP2Aa-2) (Fig. 7) that regulate plant immunity and development (Fig. 8). These results suggest that PcAvh1 is an important pathogenicity determinant in $P$. capsici, possibly by associating with plant PP2Aa isoforms.

$P c A v h l$ is rapidly induced during a very early infection stage, with the maximal expression observed at $1.5 \mathrm{hpi}$ and then declining through 3 to $24 \mathrm{hpi}$ (Fig. 1). The early, high expression level indicates that PcAvh1 is an essential virulence factor for the initial invasion and colonization of $P$. capsici. Intriguingly, PcAvhl was upregulated again at 36 and 72 hpi. This transcription profile does not perfectly fit the previously described grouping of immediate-early effectors, early effectors, or late effectors (Wang et al. 2011). P. capsici switches from biotrophy to necrotrophy at approximately 18 to 42 hpi during the infection of $N$. benthamiana (Lamour et al. 2012b). Therefore, it is possible that PcAvh1 also contributes to the establishment of necrotrophic infection. It remains obscure whether this function may be attributed to the cell death-triggering activity of PcAvh1, although the connection between the cell deathtriggering activity of PcAvh1 and its virulence role was observed (Fig. 6).

During the biotrophic infection stage (1.5 to $24 \mathrm{hpi}$ ), the cell death-inducing activity of PcAvh1 might be suppressed by 


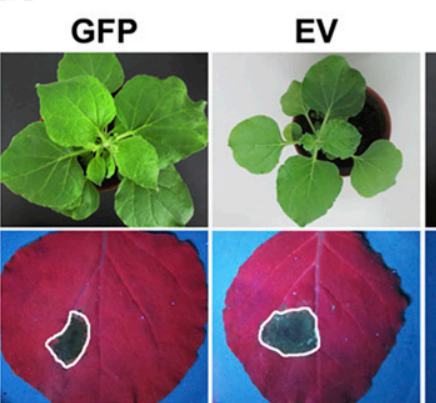

PP2A

PP2Aa-1-5' PP2Aa-1-3'

PP2Aa-2-5'

PP2Aa-2-3'

B

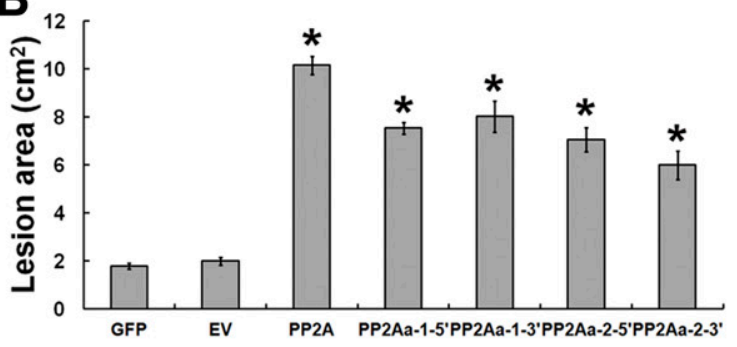

C PDS

GFP

EV

PP2A
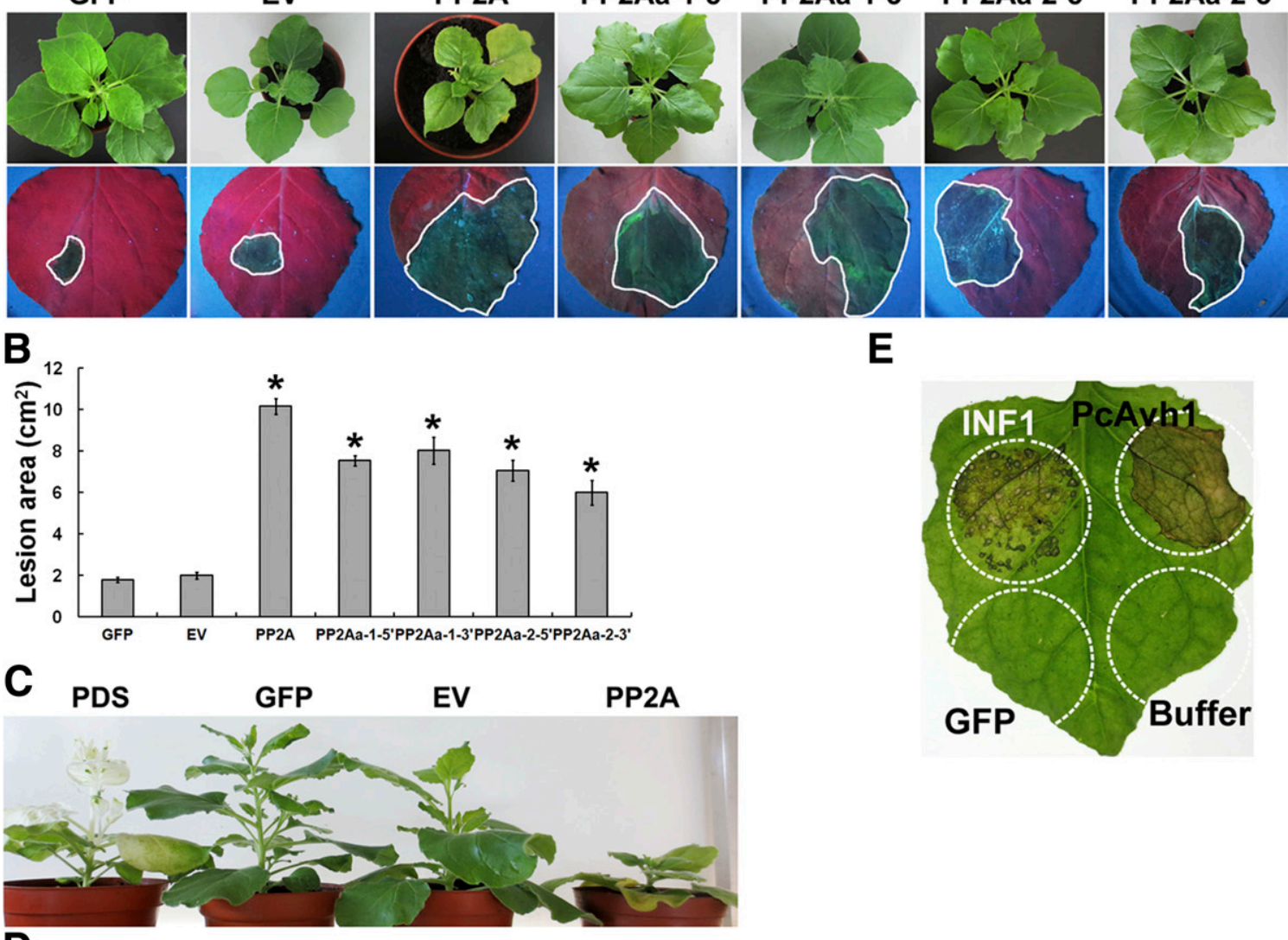

E
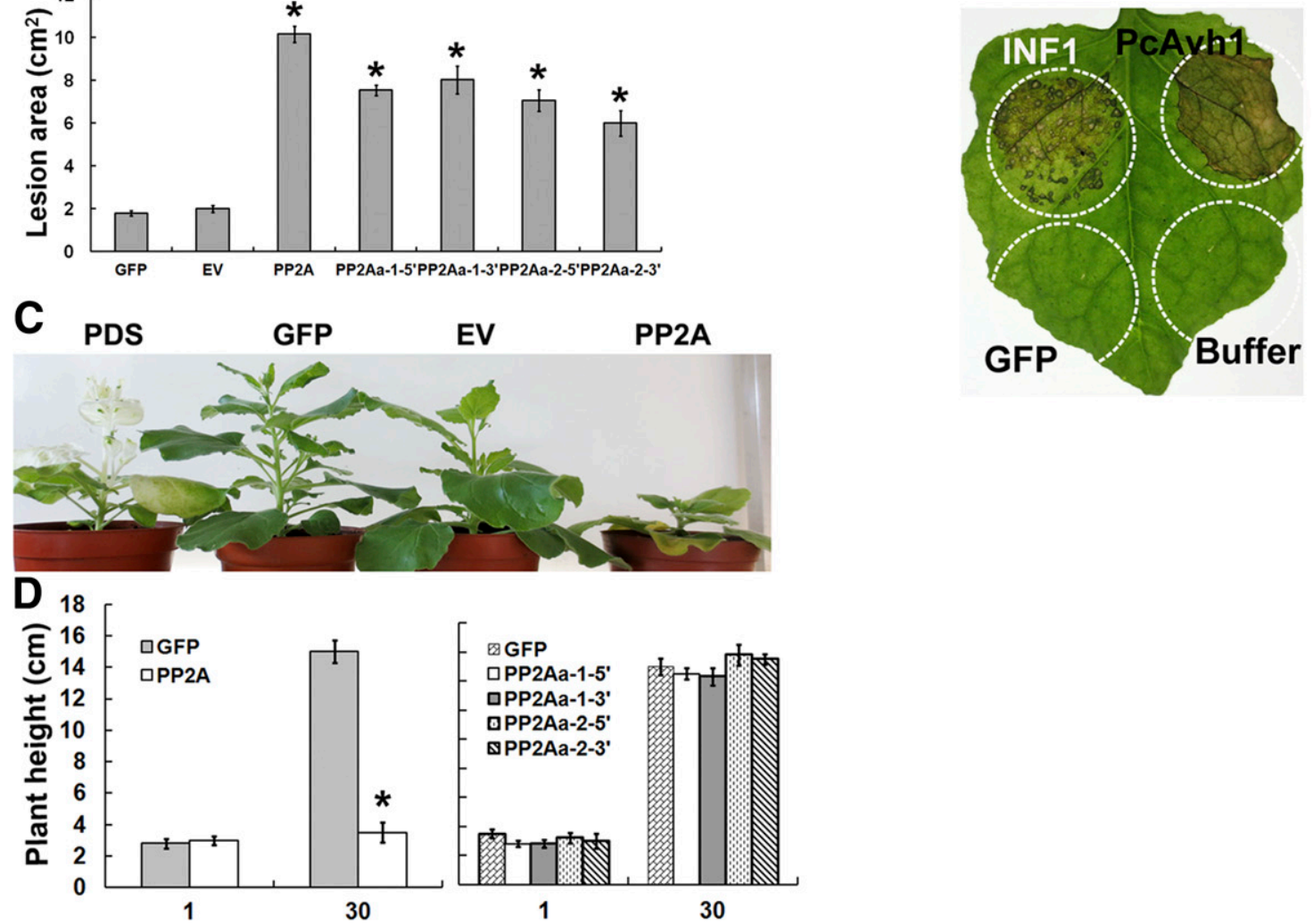

$\mathbf{F}$
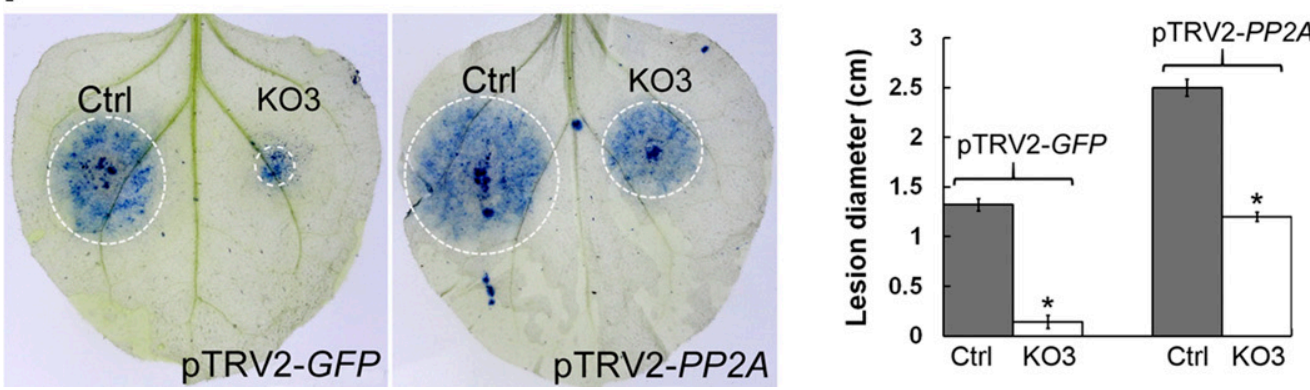

Fig. 8. NbPP2Aa-1 and NbPP2Aa-2 contribute to plant defense against Phytophthora capsici infection and also affect growth. A, Virus-induced gene silencing (VIGS) of an individual isoform gene (NbPP2Aa-1 or NbPP2Aa-2) or two isoform genes simultaneously resulted in increased $P$. capsici lesions. EV = pTRV2 alone. The irregular shaped areas indicate the lesions caused by $P$. capsici and were visualized under UV light. All VIGS plants showed a normal phenotype but the ones expressing pTRV-PP2A, which showed stunted and yellowing growth. B, Statistical analysis of lesion sizes in A. Error bars are standard error (SE) and the asterisks (*) denote statistically significant difference (Student $t$ test, $P<0.01$ ). C, Dwarfism phenotype of plants with both $N b P P 2 A a$ genes silenced. D, Reduction in plant height. Error bars are SE and the graph represents the combined data from five biological replicates $(n=50$ per construct). The asterisk (*) denotes statistically significant difference (Student $t$ test, $P<0.01$ ). E, PcAvh1 induced cell death in VIGS plants expressing pTRV2-PP2A as the positive control INF1 does at 5 days postinfiltration. The green fluorescent protein (GFP) marker and $10 \mathrm{mM} \mathrm{MgCl} 2$ buffer were used as negative controls. The dotted circles indicate the infiltration areas. F, Silencing of $P P 2 A$ genes in $N$. benthamiana compensates the virulence defect of the $P c A v h 1$ knockout. The first leaf shows the VIGS control (pTRV2-GFP), while, in the second leaf, both $N b P P 2 A a$ genes were simultaneously silenced $(\mathrm{pTRV} 2-P P 2 A)$. KO3 $=\mathrm{a}$ CRISPR/Cas9mediated PcAvhl knockout transformant, Ctrl = a non-homology-directed repair false positive transformation control. The chart shows statistical analysis of lesion size in the first and second leaves. Error bars are standard error and the asterisks $\left(^{*}\right)$ denote statistically significant difference (Student $t$ test, $P<0.01$ ). 
other effectors so that it could still contribute to pathogen infection (Chen et al. 2013). A similar example was reported in the $P$. sojae RXLR effector PsAvh238, which can suppress plant immunity to facilitate pathogen infection when its cell death-inducing activity was suppressed by other effectors produced by the same pathogen (Wang et al. 2011; Yang et al. 2017). Future work is required to further determine what roles other than PCD-inducing activity PcAvh1 may play during the infection.

Accumulating evidence suggests a link between positive selection and sequence polymorphism of effectors and shows how genes undergo accelerated birth and death evolution (Fouché et al. 2018). In this study, sequence polymorphism of PcAvh1 was observed in a collection of $31 \mathrm{P}$. capsici isolates (Fig. 2). Since the RXLR secretome is at the front line in the coevolution of the hosts and pathogens (Anderson et al. 2015; Fouché et al. 2018; Kamoun 2006), the polymorphic character of the effector suggests that the gene may play an important role in the microbe-plant interaction. However, no evidence of positive selection among the sequenced isolates was discovered. Intriguingly, evolutionary analysis suggests that PcAvhl suffered a negative selection (Table 1), especially in the Cterminus that is dedicated to the activities of effectors inside plant cells (Dou et al. 2008; Kamoun 2006; Win et al. 2007). Since a dN/dS ratio $<1$ is a hallmark of sequences that are being constrained to code for proteins, such genes can be inferred to be functional (Delport et al. 2010; Kosakovsky Pond and Frost 2005; Yang 2007). Consistent with this, the transcripts of $P c A v h l$ were detected in 1.5-hpi cDNA samples of all 31 tested isolates, meaning that the alleles were expressed during infection. We did not find any of $P$. capsici isolates with $P c A v h 1$ deletion from the test. In addition, PcAvh1 is found highly conserved across several Phytophthora species (Fig. 3), probably representing a few RXLR effectors conserved across Phytophthora species (Anderson et al. 2015; Jiang et al. 2008). Together with the gene replacement and complementation assays, all the results indicate that PcAvh1 is an important virulence factor conserved within the Phytophthora genus. Based on this, one can infer that PcAvh1 and its RXLR orthologs may target a common plant component during the infection, although further parallel investigations are required.

PP2A is a heterotrimeric Ser/Thr phosphatase that has been shown to function as a crucial coordinator of immunity in various plant species (Durian et al. 2016). The holoenzyme comprises a scaffolding and structural subunit (A), a highly variable regulatory subunit (B), and a catalytic subunit (C), with each subunit being encoded by several genes (MacKintosh and Cohen 1989). As the scaffolding subunit, the A subunit provides a protein platform that brings together the $C$ and $B$ subunit, which recruits substrate proteins for dephosphorylation. In this study, we found that NbPP2Aa interacts with PcAvh1 and contributes to plant immunity and growth in $N$. benthamiana during Phytophthora infection (Figs. 7 and 8).

The correlation of the virulence function of PcAvh1 with its targeting to host PP2Aa was tested when the inoculation of $P c A v h l \mathrm{KO}$ strains onto $P P 2 A a$ gene-silenced plants was made (Fig. 8). Silencing of PP2Aa genes in $N$. benthamiana compensates the virulence defect of the PcAvhl KO strains (Fig. 8), suggesting that PcAvh1 contributes to $P$. capsici infection, probably through its interaction with host PP2Aa. This also suggests a positive regulation role of PP2Aa in plants to defense against the $P$. capsici infection. Similarly, several studies reported the role of PP2Aa in plant defense (Jin et al. 2016; Segonzac et al. 2014). Arabidopsis RCN1, one isoform of PP2Aa, positively contributes to pathogen-induced cell-wall reinforcement and a rcnl mutant showed reduced callose deposition in response to the infection of Pseudomonas syringae pv. tomato DC3000 $\triangle$ CEL mutant, which lacks both AvrE1 and HopM1 genes (Jin et al. 2016). Alignan et al. (2006) observed an increased level of transcripts encoding PP2A (AJ539881) at $48 \mathrm{~h}$ after inoculation of sunflower with Phoma macdonaldii and presumed that the activation of PP2A could limit the propagation of the necrotrophic fungus through inhibiting localized cell death. In Arabidopsis, the A subunit and three isoforms of B subunit, B' $\eta, B^{\prime} \vartheta$, and B' $\zeta$, negatively regulate the early PTI signaling triggered by the PAMP flg22 (Jin et al. 2016). Consistently, Arabidopsis isoforms of PP2A containing $\mathrm{RCN} 1, \mathrm{~B}$ ' $\eta$ and $\mathrm{B}$ ' $\zeta$ and $\mathrm{C} 4$ subunits negatively regulate early PAMP signaling via modulating the phosphorylation status of the coreceptor BAK1 (Segonzac et al. 2014). These findings indicate that the PP2A holoenzyme and its subunits appear to have distinct impact to plant defense. There are several explanations for this, one of which is that the same subunit may have distinct functions depending on their substrates (Durian et al. 2016; Farkas et al. 2007; Jin et al. 2016). For example, mutants of the B subunit isoforms $B^{\prime} \eta, B$ ' $\vartheta$, and $B^{\prime} \zeta$ exhibited increased flg22-induced reactive oxygen species (ROS) production, while a mutation of the $B^{\prime} \alpha$ isoform led to decreased ROS production in Arabidopsis (Jin et al. 2016). In addition, the function of PP2A may be variable in different pathosystems, or, possibly, defense regulation mechanisms investigated may be different in various plant species (Durian et al. 2016; País et al. 2009).

In this study, VIGS of genes encoding two NbPP2Aa isoforms in $N$. benthamiana results in stunted plants (Fig. 8), indicating that PP2Aa regulates plant growth. This is consistent with the previous finding in Arabidopsis, which produces three isoforms of PP2Aa, namely, PDF1, PDF2, and RCN1. KO studies showed that PP2Aa is involved in phytohormone signaling and salt stress response in plants (Blakeslee et al. 2008; Farkas et al. 2007; Zhou et al. 2004). Double mutants carrying rcnl and either $p d f 1$ or $p d f 2$ exhibit severe deficiencies including dwarfing (Zhou et al. 2004), consistent with our observations in $N$. benthamiana.

So far, only a limited number of effectors have been characterized using genetic approaches, due to the intrinsic characteristics and difficulties in genetic manipulation of oomycetes (Franceschetti et al. 2017; Kamoun 2006). Remarkably, CRISPR/Cas9 technology has been recently adapted for use in $P$. sojae, which is expected to accelerate the elucidation of the pathogenicity mechanisms of oomycete pathogens (Fang and Tyler 2016; Fang et al. 2017). In this study, PcAvhl was successfully knocked out from the $P$. capsici genome by HDRmediated gene replacement using this newly developed genomeediting technology. To improve the efficiency and speed up the whole procedure, we used two sgRNA plasmids simultaneously, together with hSpCas9 and HDR donor plasmids, in the transformation process. This was proven to be feasible and efficient. We did not try more than two sgRNAs at a time but we believe it would be feasible and this strategy should be appended to the original protocol.

In summary, our work revealed an important virulence role of P. capsici RXLR effector PcAvh1 and illustrated that PcAvh1 interacts with the PP2Aa scaffolding subunit. We further demonstrated that the PP2Aa isoforms are positive regulators of plant disease resistance and growth. One $P$. infestans RXLR effector was recently shown to target host protein phosphatase 1 catalytic (PP1c) isoforms, and silencing of PP1c isoforms does not affect plant growth but attenuates infection (Boevink et al. 2016). These findings together indicate that Phytophthora pathogens may have evolved effectors to modulate host protein phosphatase pathways to their good. In this study, no counterpart R protein-encoding genes but PP2Aa were obtained from the $\mathrm{Y} 2 \mathrm{H}$ assays for PcAvh1. Expression of PcAvhl in $N b P P 2 A a$-silenced plants still causes cell death (Fig. 8E), 
indicating that cell death triggered by PcAvh1 does not depend on PP2Aa. Future work should determine the action mode of PcAvh1 and the correlation between the virulence function, cell death-inducing activity, and the interaction of PcAvh1 with PP2Aa.

\section{MATERIALS AND METHODS}

\section{Phytophthora spp. and plant cultivation.}

The Pc537 isolate of $P$. capsici originally collected from diseased bell pepper in Fujian Province, China was used in all experiments, while the other 30 isolates were exploited only for allelic sequence analysis. The cultures were maintained on $10 \%$ V8 juice agar medium, and production of cell types, including vegetative mycelia, sporangia, zoospores, and germinated cysts, was performed as previously described (Chen et al. 2013).

N. benthamiana, Solanum lycopersicum (cvs. L402, Zhongza-9, Maofen-802) (tomato) and Capsicum annuum var. grossum (bell pepper) were grown in greenhouses under a 16-h light regime at $22^{\circ} \mathrm{C}$. Two- to 6 -week-old plants were used for the following assays.

\section{Plant inoculations and trypan blue staining.}

To check the transcriptional changes of PcAvhl during infection, a set of inoculation timepoints $(1.5,3,6,12,24,36$, and 72 hpi) was established. $N$. benthamiana grown in pots were watered $3 \mathrm{~h}$ prior to rhizosphere inoculation. The plant inoculation by $P$. capsici zoospore suspension and sample harvest was performed as described (Chen et al. 2013).

To perform pathogenicity test, zoospore suspensions (100 zoospores per microliter) of the WT isolate, transformation control, and PcAvhl gene-overexpressed, -complemented, and -KO transformants were prepared to inoculate plants. The abaxial sides of detached leaves of $N$. benthamiana were inoculated with 20- $\mu$ l droplets of zoospore suspension on two spots for each leaf. Inoculated leaves, kept in trays covered with lids, were placed in $75 \%$ relative humidity and darkness at $25^{\circ} \mathrm{C}$. To better visualize the disease lesions, the inoculated leaves were stained by $0.05 \%$ trypan blue (Sigma-Aldrich, St. Louis), as described earlier (Chen et al. 2016), or were observed under a UV light. The stems of bell pepper seedlings (roots removed) were dipped into zoospore suspension for $4 \mathrm{~h}$ to get infected and were then kept under the condition described above. The pathogenicity test was biologically repeated three times with similar results.

\section{Nucleic acid manipulation and quantitative reverse transcription (qRT)-PCR.}

Genomic DNA was extracted from the Pc537 mycelia as described by Zelaya-Molina et al. (2011). RNA was isolated from Phytophthora spp. and plants using RNAiso Plus reagent (TaKaRa Biotechnology, Dalian, China), following manufacturer instructions, and was treated by DNase I (TaKaRa). Firststrand cDNA was synthesized from mRNA using M-MLV reverse transcription (RNase H Minus) and oligo (dT) 18 primers (TaKaRa) according to manufacturer suggestions.

qPCR was performed using SYBR green dye (Applied Biosystems, Foster City, CA, U.S.A.) and the gene-specific primers (Supplementary Table S2), according to manufacturer instructions. Primers were designed outside the region of cDNA targeted for silencing or $\mathrm{KO}$ following standard molecular biology guidelines. The housekeeping gene NbEF1 $\alpha$ (AY206004), encoding $N$. benthamiana elongation factor $1-\alpha$, was used as the internal control. The gene expression level was estimated by the threshold cycle values using the relative expression software tool (REST) (Pfaffl et al. 2002). The expression of each gene in the different samples was calculated relative to its expression in axenically grown mycelia. qRT-PCR reactions were biologically repeated three times and produced the similar results.

\section{Plasmid construction.}

All recombinant plasmids were validated by sequencing at GenScript Inc. (Nanjing, China). For cloning PcAvhl into binary vectors, the DNA fragments corresponding to the mature PcAvh1 protein without signal peptide were PCR-amplified from genomic DNA using high-fidelity DNA polymerase (PrimeSTAR HS DNA polymerase, TaKaRa) and specific primer pairs. To analyze cell death-inducing activity, the PcAvhl fragment was amplified using primers PcAvh1-SP-F and PcAvh1-SP-R and was directly cloned into the SmaI site of the binary vector pGR107 (Jones et al. 1999). The PcAvhl fragment was generated using primers PcAvh1-TSK-F and PcAvh1-TSK-R, with the introduction of a BamHI site at the 5' end and a NotI site at the $3^{\prime}$ end, and was subsequently cloned into the same sites of an entry vector pTsk108-N3F containing N-terminal $3 \times$ Flag, a modified variant of $p E N T R / D-T O P O$ (Invitrogen, Carlsbad, CA, U.S.A.). The entry clone insert was then transferred into pEarleyGate100 (Earley et al. 2006) by Gateway LR recombination (Invitrogen) to yield the expression clone.

To analyze the subcellular localization, PcAvhl was PCRamplified using primers PcAvh1-Bin-F and PcAvh1-Bin-R and was cloned via digesting with the SmaI and SalI restriction enzymes into the expression vector pBinGFP4 (Liu et al. 2014).

Constructs used for functional analyses of $P c A v h l$ were generated as follows. To complement and overexpress the gene, the full length of $P c A v h 1$ was amplified using primers PcAvh1TOR-F and PcAvh1-TOR-R, was cut with the $C l a \mathrm{I}$ and $B s i \mathrm{WI}$ restriction enzymes, and was inserted in sense orientation into pTORmRFP4 (Torche 2004). To accomplish HDR-mediated genome editing, a homologous donor template is provided in addition to the CRISPR/Cas9 components. The homologous donor template (namely pBS-HDR-NPTII) consisting of approximately $1 \mathrm{~kb}$ of genomic flanking sequences (homology arms) of PcAvhl on each side of the NPTII gene was assembled into pBluescript SK $\mathrm{II}^{+}$(Stratagene, La Jolla, CA, U.S.A.), following the protocol (Fang et al. 2017). Two 20-nt sgRNA oligonucleotides, 5' -AAAACCGGAACTGCTCACCT-3' (sgRNA1) and 5'-ACAAGGCGGCGAGAAATGGT-3' (sgRNA2), targeting the C-terminus of PcAvh1 were designed using the EuPaGDT web tool (Peng and Tarleton 2015), prepared as described by Fang et al. (2017), and were cloned into pYF2.3G-Ribo-sgRNA predigested by NheI and BsaI.

For the Y2H assay, the bait vector containing GAL4 DNA binding domain was prepared by cloning PcAvhl into plasmid pGBKT7 (Clontech, Mountain View, CA, U.S.A.), using primers PcAvh1-BD-F and PcAvh1-BD-R and the SalI and PstI restriction sites.

Split YFP constructs (Sun et al. 2017) were made by cloning $P c A v h 1$ into $\mathrm{p} 2 \mathrm{YC}$ to generate $\mathrm{YC}$ protein fusion and recombining $N b P P 2 A a-1$ or $N b P P 2 A a-2$ with p2YN to yield YN protein fusions.

Constructs used for in-vitro GST pull-down were prepared as follows. The PcAvhl fragment was amplified using primers PcAvh1-RSF-F and PcAvh1-RSF-R, cut by the BamHI and PstI restriction enzymes, and was cloned into similarly cleaved pRSFDuet-1 (Novagen, Madison, WI, U.S.A.) containing a $6 \times$ His-SUMO tag. For cloning the full-length coding sequences of NbPP2Aa-1 and NbPP2Aa-2, driven by the tac promoter in the plasmid pGEX-4T-2 (GE Healthcare Life Sciences, Little Chalfont, U.K.), PCR was performed on $N$. benthamiana cDNA, using primers NbPP2Aa-1-GEX-F and NbPP2Aa-1-GEX-R and 
NbPP2Aa-2-GEX-F and NbPP2Aa-2-GEX-R, respectively, containing the BamHI and SalI restriction sites.

VIGS constructs were made by cloning PCR fragments of each gene (PcAvh1, PDS [phytoene desaturase], GFP) into pTRV2 (Liu et al. 2002), between two appropriate restriction sites in antisense. Briefly, specific 120- to 750-bp fragments of the nonconserved 5' or $3^{\prime}$ regions of each PP2Aa interactor or central region shared by two isoforms were cloned into pTRV2 using different primer combinations. A 504-bp fragment of PDS (DQ469932) was amplified from $N$. benthamiana cDNA, using primers PDS-TRV-F and PDS-TRV-R. The full length of GFP from the plasmid pGFPN (Ah-Fong and Judelson 2011) was amplified using primers GFP-TRV-F and GFP-TRV-R.

\section{Agroinfection assays.}

A. tumefaciens GV3101 transformed with recombinant constructs was grown overnight in Luria-Bertani medium containing appropriate antibiotics at $28^{\circ} \mathrm{C}$, was pelleted, washed, and resuspended in infiltration buffer $[10 \mathrm{mM}$ 2-(N-morpholino) ethanesulfonic acid, $10 \mathrm{mM} \mathrm{MgCl}_{2}$, and $0.1 \mathrm{mM}$ acetosyringone], and was adjusted to the required density at $600 \mathrm{~nm}$ of 0.6 before infiltration into $N$. benthamiana, bell pepper, and tomato leaves. Agrobacteria cells were incubated in infiltration buffer for at least $2 \mathrm{~h}$ before infiltration. For cell death-inducing assays, symptoms were monitored visually at a 3 - to 15-day interval after infiltration. To coexpress multiple constructs, bacterial cultures carrying the different constructs were equally mixed prior to infiltration. The recombinant BiFC constructs were cotransformed along with the virus-silencing suppressor Tombusvirus P19 into $N$. benthamiana by A. tumefaciens infiltration, as described previously (Sun et al. 2017).

\section{Stable transformation of $\boldsymbol{P}$. capsici.}

Overexpression and complementation of $P c A v h 1$ was achieved by polyethylene glycol-mediated protoplast transformation of P. capsici (Fang et al. 2017) with the plasmid pTORmRFP4-PcAvh1. Homologous gene replacement in P. capsici was performed using the CRISPR/Cas9 system (Fang et al. 2017) with a 1:1:1 molar ratio of pYF2.3G-Ribo-sgRNA, pYF2-PsNLS-hSpCas9, and pBS-HDR-NPTII. Stable transformation was conducted as described (Fang et al. 2017) with minor modifications. Briefly, 2- to 3-day-old $P$. capsici mycelia cultured in pea broth medium were harvested for protoplast preparation. The colonies that grew well on V8 medium containing $37.5 \mu \mathrm{g}$ of G418 per milliliter were chosen for further analyses. The control transformant was transformed with the empty pTORmRFP4 vector. Putative transformants were screened by qRT-PCR, to identify overexpression or complementation lines. The HDR transformants were screened by amplifying genomic DNA of each line using different primer combinations and by NcoI digestion of PCR products as described (Fang et al. 2017). Isolation of single zoospores was performed to obtain homokaryotic mycelial colonies carrying the desired genotype.

\section{Y2H analyses.}

A mixed Y2H prey library cloned in pGADT7-Rec (Clontech) was prepared from $P$. capsici-infected leaves of $N$. benthamiana at 15 and $72 \mathrm{hpi}$, using the Make Your Own Mate \& Plate library system (Clontech). Screening of approximately $1.6 \times 10^{6}$ yeast clones with pGBKT7-PcAvhl as the bait was performed using the Matchmaker Gold Y2H system (Clontech). Interactions were confirmed using reporter gene assays, namely, ability to grow on quadruple dropout SD-4 (a SD medium [Clontech] missing histidine [-His], adenine [-Ade], leucine [-Leu], and tryptophan [-Trp]), and screening for gain of $\alpha$-galactosidase activity and aureobasidin A resistance. Candidate interacting preys were confirmed by retransformation with the PcAvh1 bait construct or with a pGBKT7 empty control. Two CaPP2Aa isoforms (XM_016692206.1, XM_016700428.1) were also cloned from bell pepper cDNA into the prey vector pGADT7 (Clontech), which were tested pairwise for the interactions with pGBKT7PcAvhl.

\section{In vitro GST pull-down assays.}

GST-fused PP2Aa proteins (both from N. benthamiana and bell pepper) and His-fused effector (PcAvh1 and 129044) proteins were expressed in E. coli Rosetta (DE3), using the pGEX-4T-2 construct and pRSFDuet-1 construct, respectively. The proteins were induced with $0.1 \mathrm{mM}$ isopropyl- $\beta$-D-thiogalactoside at $20^{\circ} \mathrm{C}$ for $12 \mathrm{~h}$. Soluble total proteins (GSTPP2Aa, GST) were incubated with $80 \mu$ l of Pierce glutathioneagarose beads (Thermo Fisher, Waltham, MA, U.S.A.) at $4^{\circ} \mathrm{C}$ for $2 \mathrm{~h}$. Then, supernatant was disposed, and the beads were washed five times with ice-cold $1 \times$ phosphate-buffered saline (PBS) buffer. Next, His-PcAvh1, His-129044, or His protein was added to the beads for the overnight incubation at $4^{\circ} \mathrm{C}$. After seven times of washing with $1 \times$ PBST (PBS with $0.1 \%$ Tween 20), the sodium dodecyl sulfate-polyacrylamide gel electrophoresis (SDS-PAGE) sample loading buffer (4\% SDS, $10 \%$ 2-mercaptoethanol, $20 \%$ glycerol, $0.004 \%$ bromophenol blue, $125 \mathrm{mM}$ Tris- $\mathrm{HCl}, \mathrm{pH} 6.8$ ) was added to beads and was heated to $100^{\circ} \mathrm{C}$ for $5 \mathrm{~min}$ for immunoblot analysis.

\section{Western blot.}

Standard SDS-PAGE electrophoresis was conducted for protein separation. Proteins were transferred from the gel to a Hybond-P polyvinylidene difluoride membrane (GE Healthcare) (pretreated with methanol for $30 \mathrm{~s}$ ) using a semiwet apparatus (Bio-Rad, Hercules, CA, U.S.A.) and a transfer buffer (25 mM Tris, $190 \mathrm{mM}$ glycine, and 20\% methanol). Then, the membrane was blocked using a blocking buffer (5\% BD Difco skim milk in Tris-buffered saline with Tween 20 (TBST) buffer (20 mM Tris, pH 7.5, $150 \mathrm{mM} \mathrm{NaCl,} \mathrm{0.1 \%} \mathrm{Tween} \mathrm{20)} \mathrm{for}$ $30 \mathrm{~min}$ at room temperature. Primary antibodies were added to new blocking buffer at a ratio of 1:2,000 and were incubated at $4^{\circ} \mathrm{C}$ overnight, followed by three washes (10 min each) with TBST. Then, the membrane was incubated with the conjugated secondary antibody at a ratio of 1:7,500 in new blocking buffer at room temperature for $2 \mathrm{~h}$. The membrane was washed three times (10 min each) with TBST. Protein bands on the membrane were detected using Thermo-Pierce ECL Western blotting substrate (Thermo Fisher).

\section{Microscopy.}

Phytophthora cells expressing GFP or monomeric red fluorescent protein (mRFP) fluorescence was detected using a BX53 fluorescence microscope with a corresponding filter and a $20 \times$ objective lens (Olympus, Tokyo). GFP was excited at $488 \mathrm{~nm}$ and its emissions were detected between 505 and $530 \mathrm{~nm}$, while mRFP was observed using $561 \mathrm{~nm}$ excitation with emissions collected between 600 and $630 \mathrm{~nm}$. At 2 dpi, GFP, split-YFP, and mCherry fluorescent signals in the tobacco epidermal cells were photographed using a Zeiss LSM 710 confocal microscope with a 20x objective lens (Zeiss Microscopy, Jena, Germany). Similarly, GFP was checked at excitation and emission wavelengths of 488 and 495 to $530 \mathrm{~nm}$, respectively. YFP and mCherry were excited at $514 \mathrm{~nm}$ and $543 \mathrm{~nm}$, respectively, and fluorescence was captured at emission wavelengths of 530 to $575 \mathrm{~nm}$ and 600 to $650 \mathrm{~nm}$, respectively.

\section{Bioinformatic analysis.}

The present assembly of $P$. capsici LT1534 genome (v11.0) is available at the Department of Energy Joint Genome Institute. 
The protein sequence was tentatively translated from the cDNA sequence of PcAvhl using BioEdit (v7.2.0). NbPP2Aa fulllength sequences were retrieved by searching (BlastN, $E \leq 1 \mathrm{e}-$ 45 ) the genomic database of $N$. benthamiana (last accessed November 5, 2015) for yeast clone sequences. The homologous representative protein sequences of NbPP2Aa in other species (rice, bell pepper, and $N$. tabacum) were retrieved from the NCBI database (last accessed September 27, 2017) by BlastP comparison $(E \leq 1 \mathrm{e}-15)$. Protein sequences of Arabidopsis PDF1, PDF2, and RCN1 (Farkas et al. 2007) were retrieved from The Arabidopsis Information Resource website (TAIR, last accessed August 8, 2016). BlastP ( $E \leq 1 \mathrm{e}-15)$ was used for homology searches of the FungiDB database, NCBI, and genome websites for several oomycetes. Sequence alignment was made using BioEdit (60\% threshold for shading).

\section{ACKNOWLEDGMENTS}

We thank Y. Fang, B. Tyler, Y. Wang, T. Liu, Q. Chen, H. Judelson, and D. Baulcombe for generously providing strains and plasmids. We also thank Y. Hou and Y. Zhao for helpful advice and generous assistance with experiments.

\section{AUTHOR-RECOMMENDED INTERNET RESOURCES}

Department of Energy Joint Genome Institute:

https://genome.jgi-psf.org/Phyca11/Phyca11.home.html

EuPaGDT: http://grna.ctegd.uga.edu

FungiDB database: http://fungidb.org

NCBI database: http://www.ncbi.nlm.nih.gov

Nicotiana benthamiana Genome and Transcriptome website:

https://sydney.edu.au/science/molecular_bioscience/sites/benthamiana

The Arabidopsis Information Resource website:

https://www.arabidopsis.org

\section{LITERATURE CITED}

Ah-Fong, A. M., and Judelson, H. S. 2011. Vectors for fluorescent protein tagging in Phytophthora: Tools for functional genomics and cell biology. Fungal Biol. 115:882-890.

Alignan, M., Hewezi, T., Petitprez, M., Dechamp-Guillaume, G., and Gentzbittel, L. 2006. A cDNA microarray approach to decipher sunflower (Helianthus annuus) responses to the necrotrophic fungus Phoma macdonaldii. New Phytol. 170:523-536.

Amaro, T. M. M. M., Thilliez, G. J. A., Motion, G. B., and Huitema, E. 2017. A perspective on CRN proteins in the genomics age: Evolution, classification, delivery and function revisited. Front. Plant Sci. 8:99.

Anderson, R. G., Deb, D., Fedkenheuer, K., and McDowell, J. M. 2015. Recent progress in RXLR effector research. Mol. Plant-Microbe Interact. 28:1063-1072.

Blakeslee, J. J., Zhou, H. W., Heath, J. T., Skottke, K. R., Barrios, J. A., Liu, S. Y., and DeLong, A. 2008. Specificity of RCN1-mediated protein phosphatase $2 \mathrm{~A}$ regulation in meristem organization and stress response in roots. Plant Physiol. 146:539-553.

Boevink, P. C., Wang, X., McLellan, H., He, Q., Naqvi, S., Armstrong, M. R., Zhang, W., Hein, I., Gilroy, E. M., Tian, Z., and Birch, P. R. 2016. A Phytophthora infestans RXLR effector targets plant PP1c isoforms that promote late blight disease. Nat. Commun. 7:10311.

Bos, J. I. B., Kanneganti, T. D., Young, C., Cakir, C., Huitema, E., Win, J., Armstrong, M. R., Birch, P. R. J., and Kamoun, S. 2006. The C-terminal half of Phytophthora infestans RXLR effector AVR3a is sufficient to trigger R3a-mediated hypersensitivity and suppress INF1-induced cell death in Nicotiana benthamiana. Plant J. 48:165-176.

Boutemy, L. S., King, S. R., Win, J., Hughes, R. K., Clarke, T. A., Blumenschein, T. M., Kamoun, S., and Banfield, M. J. 2011. Structures of Phytophthora RXLR effector proteins: A conserved but adaptable fold underpins functional diversity. J. Biol. Chem. 286: 35834-35842.

Chen, X. R., Huang, S. X., Zhang, Y., Sheng, G. L., Li, Y. P., and Zhu, F. 2018. Identification and functional analysis of the NLP-encoding genes from the phytopathogenic oomycete Phytophthora capsici. Mol. Genet. Genomics 293:931-943.

Chen, X. R., Li, Y. P., Li, Q. Y., Xing, Y. P., Liu, B. B., Tong, Y. H., and Xu, J. Y. 2016. SCR96, a small cysteine-rich secretory protein of
Phytophthora cactorum, can trigger cell death in the Solanaceae and is important for pathogenicity and oxidative stress tolerance. Mol. Plant Pathol. 17:577-587.

Chen, X. R., Xing, Y. P., Li, Y. P., Tong, Y. H., and Xu, J. Y. 2013. RNA-Seq reveals infection-related gene expression changes in Phytophthora capsici. PLoS One 8:e74588.

Couto, D., and Zipfel, C. 2016. Regulation of pattern recognition receptor signalling in plants. Nat. Rev. Immunol. 16:537-552.

Cutler, S. R., Ehrhardt, D. W., Griffitts, J. S., and Somerville, C. R. 2000. Random GFP:cDNA fusions enable visualization of subcellular structures in cells of Arabidopsis at a high frequency. Proc. Natl. Acad. Sci. U.S.A. 97:3718-3723.

Delport, W., Poon, A. F., Frost, S. D., and Kosakovsky Pond, S. L. 2010. Datamonkey 2010: A suite of phylogenetic analysis tools for evolutionary biology. Bioinformatics 26:2455-2457.

Dou, D., Kale, S. D., Wang, X., Jiang, R. H., Bruce, N. A., Arredondo, F. D., Zhang, X., and Tyler, B. M. 2008. RXLR-mediated entry of Phytophthora sojae effector Avr1b into soybean cells does not require pathogen-encoded machinery. Plant Cell 20:1930-1947.

Dou, D., and Zhou, J. M. 2012. Phytopathogen effectors subverting host immunity: Different foes, similar battleground. Cell Host Microbe 12 484-495.

Du, Y., Mpina, M. H., Birch, P. R., Bouwmeester, K., and Govers, F. 2015. Phytophthora infestans RXLR effector AVR1 interacts with exocyst component Sec5 to manipulate plant immunity. Plant Physiol. 169: 1975-1990.

Durian, G., Rahikainen, M., Alegre, S., Brosché, M., and Kangasjärvi, S. 2016. Protein phosphatase $2 \mathrm{~A}$ in the regulatory network underlying biotic stress resistance in plants. Front. Plant Sci. 7:812

Earley, K. W., Haag, J. R., Pontes, O., Opper, K., Juehne, T., Song, K., and Pikaard, C. S. 2006. Gateway-compatible vectors for plant functional genomics and proteomics. Plant J. 45:616-629.

Erwin, D. C., and Ribeiro, O. K. 1996. Phytophthora Diseases Worldwide. The American Phytopathological Society Press, St. Paul, MN, U.S.A

Fang, Y., Cui, L., Gu, B., Arredondo, F., and Tyler, B. M. 2017. Efficient genome editing in the oomycete Phytophthora sojae using CRISPR/ Cas9. Curr. Protoc. Microbiol. 44.

Fang, Y., and Tyler, B. M. 2016. Efficient disruption and replacement of an effector gene in the oomycete Phytophthora sojae using CRISPR/Cas9. Mol. Plant Pathol. 17:127-139.

Farkas, I., Dombrádi, V., Miskei, M., Szabados, L., and Koncz, C. 2007. Arabidopsis PPP family of serine/threonine phosphatases. Trends Plant Sci. 12:169-176.

Feng, B. Z., Zhu, X. P., Fu, L., Lv, R. F., Storey, D., Tooley, P., and Zhang, X. G. 2014. Characterization of necrosis-inducing NLP proteins in Phytophthora capsici. BMC Plant Biol. 14:126.

Fouché, S., Plissonneau, C., and Croll, D. 2018. The birth and death of effectors in rapidly evolving filamentous pathogen genomes. Curr. Opin. Microbiol. 46:34-42.

Franceschetti, M., Maqbool, A., Jiménez-Dalmaroni, M. J., Pennington, H. G., Kamoun, S., and Banfield, M. J. 2017. Effectors of filamentous plant pathogens: Commonalities amid diversity. Microbiol. Mol. Biol. Rev. 81:e00066-e16.

Jiang, R. H. Y., Tripathy, S., Govers, F., and Tyler, B. M. 2008. RXLR effector reservoir in two Phytophthora species is dominated by a single rapidly evolving superfamily with more than 700 members. Proc. Natl. Acad. Sci. U.S.A. 105:4874-4879.

Jin, L., Ham, J. H., Hage, R., Zhao, W., Soto-Hernández, J., Lee, S. Y., Paek, S. M., Kim, M. G., Boone, C., Coplin, D. L., and Mackey, D. 2016 Direct and indirect targeting of PP2A by conserved bacterial type-III effector proteins. PLoS Pathog. 12:e1005609.

Jones, J. D., and Dangl, J. L. 2006. The plant immune system. Nature 444: 323-329.

Jones, L., Hamilton, A. J., Voinnet, O., Thomas, C. L., Maule, A. J., and Baulcombe, D. C. 1999. RNA-DNA interactions and DNA methylation in post-transcriptional gene silencing. Plant Cell 11:2291-2301.

Kamoun, S. 2006. A catalogue of the effector secretome of plant pathogenic oomycetes. Annu. Rev. Phytopathol. 44:41-60.

Kamoun, S., Furzer, O., Jones, J. D., Judelson, H. S., Ali, G. S., Dalio, R. J., Roy, S. G., Schena, L., Zambounis, A., Panabières, F., Cahill, D., Ruocco, M., Figueiredo, A., Chen, X. R., Hulvey, J., Stam, R., Lamour, K., Gijzen, M., Tyler, B. M., Grünwald, N. J., Mukhtar, M. S., Tomé, D. F., Tör, M., Van Den Ackerveken, G., McDowell, J., Daayf, F., Fry, W. E., Lindqvist-Kreuze, H., Meijer, H. J., Petre, B., Ristaino, J., Yoshida, K., Birch, P. R., and Govers, F. 2015. The top 10 oomycete pathogens in molecular plant pathology. Mol. Plant Pathol. 16:413-434.

King, S. R. F., McLellan, H., Boevink, P. C., Armstrong, M. R., Bukharova, T., Sukarta, O., Win, J., Kamoun, S., Birch, P. R., and Banfield, M. J. 2014. 
Phytophthora infestans RXLR effector PexRD2 interacts with host MAPKKK $\varepsilon$ to suppress plant immune signaling. Plant Cell 26:1345-1359. Kong, L., Qiu, X., Kang, J., Wang, Y., Chen, H., Huang, J., Qiu, M., Zhao, Y., Kong, G., Ma, Z., Wang, Y., Ye, W., Dong, S., Ma, W., and Wang, Y. 2017. A Phytophthora effector manipulates host histone acetylation and reprograms defense gene expression to promote infection. Curr. Biol. 27:981-991.

Kosakovsky Pond, S. L., and Frost, S. D. 2005. Not so different after all: A comparison of methods for detecting amino acid sites under selection. Mol. Biol. Evol. 22:1208-1222.

Lamour, K. H., Mudge, J., Gobena, D., Hurtado-Gonzales, O. P., Schmutz, J., Kuo, A., Miller, N. A., Rice, B. J., Raffaele, S., Cano, L. M., Bharti, A. K., Donahoo, R. S., Finley, S., Huitema, E., Hulvey, J., Platt, D., Salamov, A., Savidor, A., Sharma, R., Stam, R., Storey, D., Thines, M., Win, J., Haas, B. J., Dinwiddie, D. L., Jenkins, J., Knight, J. R., Affourtit, J. P., Han, C. S., Chertkov, O., Lindquist, E. A., Detter, C., Grigoriev, I. V., Kamoun, S., and Kingsmore, S. F. 2012a. Genome sequencing and mapping reveal loss of heterozygosity as a mechanism for rapid adaptation in the vegetable pathogen Phytophthora capsici. Mol. Plant-Microbe Interact. 25:1350-1360.

Lamour, K. H., Stam, R., Jupe, J., and Huitema, E. 2012b. The oomycete broad-host-range pathogen Phytophthora capsici. Mol. Plant Pathol. 13: 329-337.

Liu, T., Song, T., Zhang, X., Yuan, H., Su, L., Li, W., Xu, J., Liu, S., Chen, L., Chen, T., Zhang, M., Gu, L., Zhang, B., and Dou, D. 2014. Unconventionally secreted effectors of two filamentous pathogens target plant salicylate biosynthesis. Nat. Commun. 5:4686.

Liu, Y., Schiff, M., Marathe, R., and Dinesh-Kumar, S. P. 2002. Tobacco Rarl, EDS1 and NPR1/NIM1 like genes are required for $N$-mediated resistance to tobacco mosaic virus. Plant J. 30:415-429.

Ma, W., and Guttman, D. S. 2008. Evolution of prokaryotic and eukaryotic virulence effectors. Curr. Opin. Plant Biol. 11:412-419.

MacKintosh, C., and Cohen, P. 1989. Identification of high levels of type 1 and type $2 \mathrm{~A}$ protein phosphatases in higher plants. Biochem. J. 262: 335-339.

Mafurah, J. J., Ma, H., Zhang, M., Xu, J., He, F., Ye, T., Shen, D., Chen, Y., Rajput, N. A., and Dou, D. 2015. A virulence essential CRN effector of Phytophthora capsici suppresses host defense and induces cell death in plant nucleus. PLoS One 10:e0127965.

País, S. M., González, M. A., Téllez-Iñón, M. T., and Capiati, D. A. 2009. Characterization of potato (Solanum tuberosum) and tomato (Solanum lycopersicum) protein phosphatases type 2A catalytic subunits and their involvement in stress responses. Planta 230:13-25.

Peng, D., and Tarleton, R. 2015. EuPaGDT: A web tool tailored to design CRISPR guide RNAs for eukaryotic pathogens. Microb. Genom. 1: e000033.

Pfaffl, M. W., Horgan, G. W., and Dempfle, L. 2002. Relative expression software tool (REST) for group-wise comparison and statistical analysis of relative expression results in real-time PCR. Nucleic Acids Res. 30:e36.

Qiao, Y., Shi, J., Zhai, Y., Hou, Y., and Ma, W. 2015. Phytophthora effector targets a novel component of small RNA pathway in plants to promote infection. Proc. Natl. Acad. Sci. U.S.A. 112:5850-5855.

Segonzac, C., Macho, A. P., Sanmartín, M., Ntoukakis, V., SánchezSerrano, J. J., and Zipfel, C. 2014. Negative control of BAK1 by protein phosphatase 2A during plant innate immunity. EMBO J. 33:2069-2079.
Stam, R., Howden, A. J. M., Delgado-Cerezo, M., Amaro, T. M. M. M., Motion, G. B., Pham, J., and Huitema, E. 2013a. Characterization of cell death inducing Phytophthora capsici CRN effectors suggests diverse activities in the host nucleus. Front. Plant Sci. 4:387.

Stam, R., Jupe, J., Howden, A. J. M., Morris, J. A., Boevink, P. C., Hedley, P. E., and Huitema, E. 2013b. Identification and characterization CRN effectors in Phytophthora capsici shows modularity and functional diversity. PLoS One 8:e59517.

Sun, J., Zheng, T., Yu, J., Wu, T., Wang, X., Chen, G., Tian, Y., Zhang, H., Wang, Y., Terzaghi, W., Wang, C., and Wan, J. 2017. TSV, a putative plastidic oxidoreductase, protects rice chloroplasts from cold stress during development by interacting with plastidic thioredoxin Z. New Phytol. 215:240-255.

Torche, S. 2004. Isolation et etudes d'expression de genes potentiellement lis a la pathogenicite chez Phytophthora porri. Ph.D. thesis. University of Friborg, Switzerland.

Vega-Arreguín, J. C., Jalloh, A., Bos, J. I., and Moffett, P. 2014 Recognition of an Avr3a homologue plays a major role in mediating nonhost resistance to Phytophthora capsici in Nicotiana species. Mol. Plant-Microbe Interact. 27:770-780.

Wang, Q., Han, C., Ferreira, A. O., Yu, X., Ye, W., Tripathy, S., Kale, S. D., Gu, B., Sheng, Y., Sui, Y., Wang, X., Zhang, Z., Cheng, B., Dong, S., Shan, W., Zheng, X., Dou, D., Tyler, B. M., and Wang, Y. 2011. Transcriptional programming and functional interactions within the Phytophthora sojae RXLR effector repertoire. Plant Cell 23:20642086.

Wang, Y., and Wang, Y. 2018. Trick or treat: Microbial pathogens evolved apoplastic effectors modulating plant susceptibility to infection. Mol. Plant-Microbe Interact. 31:6-12.

Win, J., Morgan, W., Bos, J., Krasileva, K. V., Cano, L. M., ChaparroGarcia, A., Ammar, R., Staskawicz, B. J., and Kamoun, S. 2007. Adaptive evolution has targeted the C-terminal domain of the RXLR effectors of plant pathogenic oomycetes. Plant Cell 19:2349-2369.

Yang, B., Wang, Q., Jing, M., Guo, B., Wu, J., Wang, H., Wang, Y., Lin, L., Wang, Y., Ye, W., Dong, S., and Wang, Y. 2017. Distinct regions of the Phytophthora essential effector Avh238 determine its function in cell death activation and plant immunity suppression. New Phytol. 214: 361-375.

Yang, L., McLellan, H., Naqvi, S., He, Q., Boevink, P. C., Armstrong, M., Giuliani, L. M., Zhang, W., Tian, Z., Zhan, J., Gilroy, E. M., and Birch, P. R. 2016. Potato NPH3/RPT2-like protein StNRL1, targeted by a Phytophthora infestans RXLR effector, is a susceptibility factor. Plant Physiol. 171:645-657.

Yang, Z. 2007. PAML 4: Phylogenetic analysis by maximum likelihood. Mol. Biol. Evol. 24:1586-1591.

Ye, W., and Ma, W. 2016. Filamentous pathogen effectors interfering with small RNA silencing in plant hosts. Curr. Opin. Microbiol. 32:1-6.

Zelaya-Molina, L. X., Ortega, M. A., and Dorrance, A. E. 2011. Easy and efficient protocol for oomycete DNA extraction suitable for population genetic analysis. Biotechnol. Lett. 33:715-720.

Zhou, H. W., Nussbaumer, C., Chao, Y., and DeLong, A. 2004. Disparate roles for the regulatory A subunit isoforms in Arabidopsis protein phosphatase 2A. Plant Cell 16:709-722. 